\title{
Seismic gravity-gradient noise in interferometric gravitational-wave detectors
}

\author{
Scott A. Hughes \\ Theoretical Astrophysics, California Institute of Technology, Pasadena, California 91125 \\ Kip S. Thorne \\ Theoretical Astrophysics, California Institute of Technology, Pasadena, California 91125 \\ and Max-Planck-Institut für Gravitationsphysik, Schlatzweg 1, 14473 Potsdam, Germany
}

(Received 4 June 1998; published 18 November 1998)

\begin{abstract}
When ambient seismic waves pass near and under an interferometric gravitational-wave detector, they induce density perturbations in the Earth, which in turn produce fluctuating gravitational forces on the interferometer's test masses. These forces mimic a stochastic background of gravitational waves and thus constitute a noise source. This seismic gravity-gradient noise has been estimated and discussed previously by Saulson using a simple model of the Earth's ambient seismic motions. In this paper, we develop a more sophisticated model of these motions, based on the theory of multimode Rayleigh and Love waves propagating in a multilayer medium that approximates the geological strata at the LIGO sites, and we use this model to reexamine seismic gravity gradients. We characterize the seismic gravity-gradient noise by a transfer function, $T(f) \equiv \widetilde{x}(f) / \widetilde{W}(f)$, from the spectrum of rms seismic displacements averaged over vertical and horizontal directions, $\widetilde{W}(f)$, to the spectrum of interferometric test-mass motions, $\widetilde{x}(f) \equiv L \widetilde{h}(f)$; here $L$ is the interferometer arm length, $\widetilde{h}(f)$ is the gravitational-wave noise spectrum, and $f$ is frequency. Our model predicts a transfer function with essentially the same functional form as that derived by Saulson, $T$ $\simeq 4 \pi G \rho(2 \pi f)^{-2} \beta(f)$, where $\rho$ is the density of Earth near the test masses, $G$ is Newton's constant, and $\beta(f) \equiv \gamma(f) \Gamma(f) \beta^{\prime}(f)$ is a dimensionless reduced transfer function whose components $\gamma \simeq 1$ and $\Gamma \simeq 1$ account for a weak correlation between the interferometer's two corner test masses and a slight reduction of the noise due to the height of the test masses above the Earth's surface. This paper's primary foci are (i) a study of how $\beta^{\prime}(f) \simeq \beta(f)$ depends on the various Rayleigh and Love modes that are present in the seismic spectrum, (ii) an attempt to estimate which modes are actually present at the two LIGO sites at quiet times and at noisy times, and (iii) a corresponding estimate of the magnitude of $\beta^{\prime}(f)$ at quiet and noisy times. We conclude that at quiet times $\beta^{\prime} \simeq 0.35-0.6$ at the LIGO sites, and at noisy times $\beta^{\prime} \simeq 0.15-1.4$. (For comparison, Saulson's simple model gave $\beta=\beta^{\prime}=1 / \sqrt{3}=0.58$.) By folding our resulting transfer function into the "standard LIGO seismic spectrum," which approximates $\tilde{W}(f)$ at typical times, we obtain the gravitygradient noise spectra. At quiet times this noise is below the benchmark noise level of "advanced LIGO interferometers" at all frequencies (though not by much at $\sim 10 \mathrm{~Hz}$ ); at noisy times it may significantly exceed the advanced noise level near $10 \mathrm{~Hz}$. The lower edge of our quiet-time noise constitutes a limit, beyond which there would be little gain from further improvements in vibration isolation and thermal noise, unless one can also reduce the seismic gravity gradient noise. Two methods of such reduction are briefly discussed: monitoring the Earth's density perturbations near each test mass, computing the gravitational forces they produce, and correcting the data for those forces; and constructing narrow moats around the interferometers' corner and end stations to shield out the fundamental-mode Rayleigh waves, which we suspect dominate at quiet times. [S0556-2821(98)00424-X]
\end{abstract}

PACS number(s): 04.80.Nn

\section{INTRODUCTION AND SUMMARY}

Now that the Laser Interferometric Gravitational Wave Observatory (LIGO) and VIRGO international network of gravitational-wave detectors [1-4] is under construction, it is important to reexamine the various noise sources that will constrain the network's ultimate performance. Improved estimates of the ultimate noise spectra are a foundation for long-term planning on a number of aspects of gravitationalwave research, including facilities design, interferometer research and design, data analysis algorithm development, and astrophysical source studies.

In this paper and a subsequent one [5] we reexamine gravity-gradient noise-noise due to fluctuating Newtonian gravitational forces that induce motions in the test masses of an interferometric gravitational-wave detector. Gravity gra- dients are potentially important at the low end of the interferometers' frequency range, $f \leqq 20 \mathrm{~Hz}$. Another noise source that is important at these frequencies is vibrational seismic noise, in which the ground's ambient motions, filtered through the detector's vibration isolation system, produce motions of the test masses. It should be possible and practical to isolate the test masses from these seismic vibrations down to frequencies as low as $f \sim 3 \mathrm{~Hz}$ [6], but it does not look practical to achieve large amounts of isolation from the fluctuating gravity gradients. Thus, gravity gradients constitute an ultimate low-frequency noise source; seismic vibrations do not.

Gravity gradients were first identified as a potential noise source in interferometric gravitational-wave detectors by Weiss in 1972 [7]. The first quantitative analyses of such gravity-gradient noise were performed by Saulson [8] and 
Spero [9] in the early 1980s. There has been little further study of gravity-gradient noise since then, except for some updating in Saulson's recent monograph [10].

In his updating, Saulson concluded that the most serious source of gravity-gradient noise will be the fluctuating density of the earth beneath and near each of the interferometer's test masses. These density fluctuations are induced by ambient seismic waves that are always present; their resulting gravitational forces are called seismic gravity-gradient noise. ${ }^{1}$ Saulson $[8,10]$ also estimated the gravity gradient noise from atmospheric fluctuations, concluding that it is probably weaker than that from earth motions. Spero [9] showed that gravity-gradient noise due to jerky human activity (and that of dogs, cattle, and other moving bodies) can be more serious than seismic gravity-gradient noise if such bodies are not kept at an adequate distance from the test masses. We shall revisit seismic gravity-gradient noise in this paper, and gravity gradients due to human activity in a subsequent one [5]; T. Creighton at Caltech has recently initiated a careful revisit of gravity gradient noise due to atmospheric fluctuations.

Our detailed analysis in this paper reveals a level of seismic gravity-gradient noise that agrees remarkably well with Saulson's much cruder estimates. Our analysis reveals the uncertainties in the gravity gradient noise, the range in which the noise may vary from seismically quiet times to noisy times, the dependence of the noise on the various seismic modes that are excited, and the characteristics of the modes that the geological strata at Hanford and Livingston are likely to support. The dependence of the noise on the modes and the characteristics of the expected modes are potential foundations for methods of mitigating the seismic gravity gradient noise, discussed in our concluding section.

A preliminary version of this paper [12] was circulated to the gravitational-wave-detection community in 1996. That version considered only fundamental-mode Rayleigh waves (which we suspect are responsible for the dominant seismic gravity-gradient noise at quiet times), and (as Ken Libbrecht pointed out to us) it contained a serious error: the omission of the "surface-source" term [denoted $\xi_{V}$ in Eq. (1.24) below] for the gravity-gradient force. It also contained errors in its two-geological-layer analysis for the LIGO Hanford site. These errors have been corrected in this final version of the manuscript, and the analysis has been extended to include more realistic models of the geological strata at the two LIGO sites and to include higher-order seismic modes.

As we were completing this manuscript, we learned of a paper in press [11] by Giancarlo Cella, Elena Cuoco and their VIRGO-Project collaborators, which also analyzes seismic gravity-gradient noise in interferometric gravitational wave detectors. That paper is complementary to ours. Both papers analyze the RF mode (which we suspect is the domi-

\footnotetext{
${ }^{1}$ Although widely used, the name " gravity-gradient noise" is a bit of a misnomer: it is not gradients of the local gravitational field which cause this noise, but simply the direct fluctuation of the gravitational force. Some other authors have used the term "Newtonian noise", [11] or "local gravitational noise" [10].
}

nant contributor to the seismic gravity-gradient noise at quiet times), obtaining the same results in the $3-30 \mathrm{~Hz}$ band when the effects of geological stratification are neglected. But, whereas our paper carries out an extensive study of the effects of stratification and other modes, the Cella-Cuoco paper extends the unstratified RF-mode analysis to frequencies below $3 \mathrm{~Hz}$ and above $30 \mathrm{~Hz}$, and computes (and finds to be small) the gravity gradient noise caused by seismicallyinduced motions of the experimental apparatus and its massive physical infrastructure in the vicinity of the VIRGO test masses.

This paper is organized as follows: In Sec. I A, we describe the phenomenology of the seismic-wave modes that can contribute to ambient earth motions at horizontally stratified sites like LIGO-Hanford and LIGO-Livingston. In Sec. I B, we introduce the transfer function $T(f)$ used to characterize seismic gravity-gradient noise, we break it down into its components [most especially the reduced transfer function $\left.\beta^{\prime}(f)\right]$, and we express it as an incoherent sum over contributions from the various seismic modes. In Sec. IC, we briefly describe Saulson's computation of the reduced transfer function, and then in Sec. I D we describe our own computation and results. More specifically, in I D we gather together and summarize from the body of the paper our principal conclusions about $\beta^{\prime}$ for the various modes at the two LIGO sites, we discuss the evidence as to which modes actually contribute to the noise at quiet times and at noisy times, and we therefrom estimate the net values of $\beta^{\prime}$ at quiet and noisy times. We then fold those estimates into the standard LIGO seismic spectrum to get spectral estimates of the seismic gravity-gradient noise (Fig. 2).

The remainder of the paper (summarized just before the beginning of Sec. II) presents our detailed models for the geological strata at the two LIGO sites, and our analyses of the various seismic modes that those strata can support and of the seismic gravity-gradient noise produced by each of those modes.

\section{A. Phenomenology of ambient seismic motions in the LIGO frequency band}

Seismic motions are conventionally decomposed into two components [13-16]: P-waves and $S$-waves. $\mathrm{P}$-waves have material displacements along the propagation direction, a restoring force due to longitudinal stress (pressure-hence the name P-waves), and a propagation speed determined by the material's density $\rho$ and bulk and shear moduli $K$ and $\mu$ :

$$
c_{P}=\sqrt{\frac{K+4 \mu / 3}{\rho}} .
$$

S-waves have transverse displacements, restoring force due to shear stress, and propagation speed

$$
c_{S}=\sqrt{\frac{\mu}{\rho}}=\sqrt{\frac{1-2 \nu}{2-2 \nu}} c_{P} \sim \frac{c_{P}}{2} .
$$

Here $\nu$ is the material's Poisson ratio 


$$
\nu=\frac{3 K-2 \mu}{2(3 K+\mu)} .
$$

Near the earth's surface, where seismic gravity-gradient noise is generated, these speeds are in the range $c_{P}$ $\sim 500-2000 \mathrm{~m} / \mathrm{s}$ and $c_{S} \sim 250-700 \mathrm{~m} / \mathrm{s}$. However, some of the modes that may contribute to the noise extend down to much greater depths, even into the bedrock where $c_{P}$ $\sim 5000-6000 \mathrm{~m} / \mathrm{s}$ and $c_{S} \sim 3200 \mathrm{~m} / \mathrm{s}$.

The ambient seismic motions are a mixture of $\mathrm{P}$-waves and S-waves that propagate horizontally ("surface waves"), confined near the earth's surface by horizontal geological strata. Depending on the mode type and frequency, the horizontal propagation speed $c_{H}$ can range from the surface layers' lowest S-speed to the bedrock's highest P-speed: $250 \mathrm{~m} / \mathrm{s} \lesssim c_{H} \lesssim 6000 \mathrm{~m} / \mathrm{s}$.

$\mathrm{P}$ - and S-waves are coupled by geological inhomogeneities (typically discontinuities at geological strata) and by a boundary condition at the earth's surface. At both LIGO sites the strata are alluvial deposits above bedrock, with discontinuities that are horizontal to within 2 degrees (more typically to within less than 1 degree). Throughout this paper we shall approximate the material as precisely horizontally stratified.

Seismic gravity-gradient noise is a potentially serious issue in the frequency band from $f \sim 3 \mathrm{~Hz}$ (the lowest frequency at which mechanical seismic isolation looks practical) to $f \sim 30 \mathrm{~Hz}$; $c f$. Fig. 2 below. In this frequency band, the wavelengths of $\mathrm{P}$ - and $\mathrm{S}$-waves are

$$
\begin{aligned}
& \lambda_{P}=100 \mathrm{~m} \frac{\left(c_{P} / 1000 \mathrm{~ms}^{-1}\right)}{(f / 10 \mathrm{~Hz})}, \\
& \lambda_{S}=50 \mathrm{~m} \frac{\left(c_{S} / 500 \mathrm{~ms}^{-1}\right)}{(f / 10 \mathrm{~Hz})} .
\end{aligned}
$$

Neglecting coupling, the amplitudes of these waves attenuate as $\exp (-\pi r / Q \lambda)$, where $r$ is the distance the waves have propagated and $Q$ is the waves' quality factor. The dominant dissipation is produced by the waves' shear motions and can be thought of as arising from an imaginary part of the shear modulus in expressions (1.1) and (1.2) for the propagation speeds $c_{S}$ and $c_{P}$ (and thence also from an imaginary part of the propagation speeds themselves). Since the restoring force for $\mathrm{S}$-waves is entirely due to shear, and for P-waves only about half due to shear, the S-waves attenuate about twice as strongly as the P-waves. The measured $Q$-factors for near-surface materials are $Q_{S} \sim 10-25, Q_{P}$ $\sim 20-50[17,18]$, corresponding to amplitude attenuation lengths

$$
\begin{gathered}
\mathcal{L}_{P}=\frac{Q_{P} \lambda_{P}}{\pi}=1000 \mathrm{~m} \frac{\left(Q_{P} / 30\right)\left(c_{P} / 1000 \mathrm{~ms}^{-1}\right)}{f / 10 \mathrm{~Hz}}, \\
\mathcal{L}_{S}=\frac{Q_{S} \lambda_{S}}{\pi}=250 \mathrm{~m} \frac{\left(Q_{S} / 15\right)\left(c_{S} / 500 \mathrm{~ms}^{-1}\right)}{f / 10 \mathrm{~Hz}} .
\end{gathered}
$$

For bedrock (and basalt that overlies it at Hanford), the $Q$ 's and attenuation lengths can be higher than this $-Q_{P}$ as high as a few hundred [19].
Shallowly seated wave modes which cause ambient seismic motions in our band, i.e., modes that are confined to the alluvia so $c_{H} \lesssim 2500 \mathrm{~m} / \mathrm{s}$ (and more typically $\lesssim 1000 \mathrm{~m} / \mathrm{s}$ ), must be generated in the vicinity of the interferometers' corner and end stations by surface sources such as wind, rain, and human activities (automobile traffic, sound waves from airplanes, etc.); their attenuation lengths are too short to be generated from further than a kilometer or so. Deep seated modes that reach into the bedrock could originate from rather further away - at $10 \mathrm{~Hz}$ and in a layer that has $Q_{P} \sim 100$, $c_{P} \sim 5500 \mathrm{~m} / \mathrm{s}$, modes can propagate as far as $\sim 20 \mathrm{~km}$.

In horizontally stratified material, the wave components that make up each mode all propagate with the same angular frequency $\omega=2 \pi f$, horizontal wave vector $\vec{k}=k \hat{k}$ (where $\hat{k}$ is their horizontal direction, and $k=2 \pi / \lambda$ their horizontal wavenumber), and horizontal phase speed $c_{H}=\omega / k$. Their vertical motions differ from one horizontal layer to another and from P-component to S-component. The horizontal dispersion relation $\omega(k)$ [or equivalently $c_{H}(f)$ ] depends on the mode (Figs. 5, 6, 9, and 11 below).

Geophysicists divide these surface normal modes into two types $[15,16]$ :

Love modes, which we shall denote by L. These are Swaves with horizontal displacements ("SH-waves") that resonate in the near-surface strata. They involve no P-waves and thus have no compression and no density variations; therefore, they produce no fluctuating gravitational fields and no seismic gravity-gradient noise.

Rayleigh modes, which we shall denote by $\mathrm{R}$. These are combinations of S-waves with vertical displacements and $\mathrm{P}$ waves ("SV-waves") that are coupled by the horizontal discontinuities at strata interfaces, including the earth's surface. Rayleigh modes are the producers of seismic gravitygradient noise.

We shall divide the Rayleigh modes into two groups: the fundamental Rayleigh mode, which we denote RF, and Rayleigh overtones (all the other modes). Rayleigh overtones require stratification of the geological structure in order to be present; they essentially consist of coupled SV- and P-waves which bounce and resonate between the earth's surface and the interfaces between strata. We shall further divide the Rayleigh overtones into two broad classes: those that are composed predominantly of SV-waves, denoted RS, and those composed predominantly of P-waves, denoted RP. In the geophysics literature, the modes we identify as RP are sometimes referred to simply as P-modes, and our RS modes are referred to as the Rayleigh overtones. However, when RP modes are intermixed with $\mathrm{RS}$ modes in the $\left(c_{H}, f\right)$ space of dispersion relations (as turns out to be the case at Hanford; cf. Fig. 6 below), a given Rayleigh overtone will continuously change character from RS to RP. Because this will be quite important for the details of the seismic gravity-gradient noise, we prefer to emphasize the similarities of the two mode types by designating them both as Rayleigh overtones and denoting them RS and RP.

We shall append to each Rayleigh overtone an integer that identifies its order in increasing horizontal speed $c_{H}$ at fixed frequency $f$. Each successive Rayleigh mode, RF, RS1, RS2, ... (and, as a separate series, RF, RP1, RP2, ...) penetrates 
more deeply into the Earth than the previous one. In our frequency band, the fundamental RF is typically confined to within $\sim \lambda_{S} / \pi \sim 10 \mathrm{~m}$ of the earth's surface.

The RF mode is evanescent in all layers (except, at low frequencies, in the top layer). The overtones RS1, RS2, ... are composed primarily of SV-waves that propagate downward from the Earth's surface, reflect off some interface, return to the surface and reflect back downward in phase with the original downward propagating waves, thereby guaranteeing resonance. On each reflection and at each interface between layers, these modes generate a non-negligible admixture of P-waves. The RP overtones are similar to RS, but with the propagating and reflecting waves being largely $\mathrm{P}$ with some non-negligible accompanying SV.

Dissipation will cause an overtone's waves to damp out with depth. If that damping is substantial in traveling from the surface to the reflection point, the overtone will not resonate and will be hard to excite. Roughly speaking, the amount of amplitude decay in traveling from the surface to the reflection point and back to the surface is $n \pi / Q$ where $n$ is the mode number (or equivalently the number of roundtrip wavelengths); cf. Eqs. (1.5). The round-trip damping therefore exceeds $1 / e$ for mode numbers $n \geqslant Q_{S} / \pi \sim 5$ for RS modes and $n \geqslant Q_{P} / \pi \sim 10$ for RP modes. Correspondingly, in this paper we shall confine attention to modes with mode numbers $n \lesssim 10$.

The RP modes are harder to analyze with our formalism than RS modes-typically, when RP modes turn on, there are many modes very closely spaced together and it is difficult to distinguish them. For this reason, we shall study only the lowest one at each site, RP1, plus RP modes that travel nearly horizontally in the several-km thick basalt layer at Hanford. We expect RP1 to be typical of other low-order RP modes, and the basalt-layer RP waves to be typical also of such waves propagating nearly horizontally in the bedrock.

\section{B. Transfer functions and anisotropy ratio}

Following Saulson [10], we shall embody the results of our gravity-gradient analysis in a transfer function

$$
T(f) \equiv \frac{\widetilde{x}(f)}{\widetilde{W}(f)}
$$

from seismic-induced earth motions $\widetilde{W}(f)$ to differential test-mass motion $\widetilde{x}(f)$. The precise definitions of $\widetilde{W}(f)$ and $\widetilde{x}(f)$ are as follows:

We shall denote the square root of the spectral density (the "spectrum") of the earth's horizontal surface displacements along some arbitrary horizontal direction by $\widetilde{X}(f)$ (units $\mathrm{m} / \sqrt{\mathrm{Hz}}$ ), where $f$ is frequency. We assume that $\widetilde{X}(f)$ is independent of the chosen direction, i.e. the seismic motions are horizontally isotropic. This is justified by seismometer measurements at the LIGO sites before construction began $[20,21]$ and by rough estimates of the diffractive influence of the constructed facilities (Sec. V). We shall denote the spectrum of vertical displacements at the earth's surface by $\widetilde{Z}(f)$. The quantity $\widetilde{W}(f)$ that appears in the transfer function is the displacement rms-averaged over 3dimensional directions:

$$
\widetilde{W}(f)=\sqrt{\frac{2 \widetilde{X}^{2}(f)+\widetilde{Z}^{2}(f)}{3}} .
$$

The other quantity, $\widetilde{x}(f)$, which appears in the transfer function (1.6) is related to the interferometer's gravitationalwave strain noise spectrum $\widetilde{h}(f)$ by $\widetilde{x}(f) \equiv \widetilde{h}(f) L$, where $L$ is the interferometer arm length (4 km for LIGO). Physically, $\widetilde{x}(f)$ is the spectrum of the interferometer's armlength difference and is called the interferometer's "displacement noise spectrum." Since $\widetilde{x}(f)$ and $\widetilde{W}(f)$ both have units of $\mathrm{m} / \sqrt{\mathrm{Hz}}$, the transfer function $T(f)$ is dimensionless.

In this paper we shall express $T(f)$ in terms of a dimensionless correction $\beta(f)$ to a simple and elegant formula that Saulson [8] derived:

$$
\begin{aligned}
T(f) & \equiv \frac{\widetilde{x}(f)}{\widetilde{W}(f)} \\
= & \frac{4 \pi G \rho}{\sqrt{\left(\omega^{2}-\omega_{0}^{2}\right)^{2}+\omega^{2} / \tau^{2}}} \beta(f) \simeq \frac{4 \pi G \rho}{(2 \pi f)^{2}} \beta(f) \\
& \text { at } f \gtrsim 3 \mathrm{~Hz} .
\end{aligned}
$$

Here $\rho \simeq 1.8 \mathrm{~g} / \mathrm{cm}^{3}$ is the mass density of the earth in the vicinity of the interferometer, $G$ is Newton's gravitational constant, $\omega=2 \pi f$ is the angular frequency of the seismic waves and their fluctuating gravitational forces, and $\omega_{0}$ $\sim 2 \pi \mathrm{rad} / \mathrm{s}$ and $\tau \sim 10^{8} \mathrm{~s}$ are the angular frequency and damping time of the test mass's pendular swing. We shall call $\beta(f)$ the reduced transfer function. Saulson's estimate for $\beta(f)$ was

$$
\beta_{\text {Saulson }}=1 / \sqrt{3}=0.58
$$

cf. Eq. (21) of Ref. [8]. Our analyses (below) suggest that at quiet times $\beta$ may be $\simeq 0.35$ to 0.6 , and at noisy times, $\beta$ $\simeq 0.15$ to 1.4 . Thus, Saulson's rough estimate was remarkably good.

Each mode of the earth's motion will contribute to the transfer function, and since the relative phases of the modes should be uncorrelated, they will contribute to $\beta(f)$ in quadrature:

$$
\beta=\sqrt{\sum_{J} w_{J} \beta_{J}^{2}}
$$

The sum runs over all Rayleigh and Love modes, $J$ $\in(\mathrm{RF}, \mathrm{RS} n, \mathrm{RP} n, \mathrm{~L} n) ; \beta_{J}(f)$ is the reduced transfer function for mode $J$, with

$$
\beta_{L n}=0
$$

because the Love modes produce no gravity-gradient noise. The weighting factor $w_{J}$ is the fractional contribution of 
mode $J$ to the mean square seismic displacement $\widetilde{W}^{2}$, and correspondingly the $w_{J}$ 's are normalized by

$$
\sum_{J} w_{J}=1
$$

Besides this normalization condition, there is another constraint on the weighting factors $w_{J}$ : each mode (at each frequency) has its own ratio $\mathcal{A}_{J}$ of vertical to horizontal displacement at the Earth's surface:

$$
\mathcal{A}_{J}(f)=\frac{\widetilde{Z}_{J}(f)}{\widetilde{X}_{J}(f)} .
$$

We shall call this ratio the mode's anisotropy ratio. ${ }^{2}$ Since the Love modes have purely horizontal motions, their anisotropy ratios vanish:

$$
\mathcal{A}_{L n}=0 \text {. }
$$

It is straightforward to show that the anisotropy ratios for the various modes combine to produce the following net anisotropy in the earth's surface displacement:

$$
\mathcal{A} \equiv \frac{\widetilde{Z}}{\widetilde{X}}=\frac{\sqrt{\sum_{J} w_{J} \mathcal{A}_{J}^{2} /\left(2+\mathcal{A}_{J}^{2}\right)}}{\sqrt{\sum_{J} w_{J} /\left(2+\mathcal{A}_{J}^{2}\right)}} .
$$

At quiet times, measurements show this to be near unity at Hanford [21], and $\sim 0.6$ at Livingston [20], while at noisy times it can fluctuate from $\sim 0.2$ to $\sim 5$. The measured value of this ratio is an important constraint on the mixture of modes that produces the observed seismic noise and thence on the net reduced transfer function. For example, if the observed noise is due to one specific Rayleigh mode $J$ with large anisotropy ratio $\mathcal{A}_{J}$, accompanied by enough Love waves to reduce the net anisotropy ratio to $\mathcal{A}_{\text {net }}=1.0$ (Hanford) or 0.6 (Livingston), then Eqs. (1.10)-(1.15) imply that the net reduced transfer function for the seismic gravity gradient noise is

$$
\beta_{J L}=\beta_{J} \sqrt{\frac{1+2 / \mathcal{A}_{J}^{2}}{1+2 / \mathcal{A}_{\mathrm{net}}^{2}}}
$$

In Appendix $\mathrm{A}$ it is shown that for each mode $J$, the reduced transfer function $\beta_{J}$ can be split into the product of three terms:

$$
\beta_{J}=\gamma_{J} \Gamma_{J} \beta_{J}^{\prime}
$$

The first term, $\gamma_{J}$, accounts for the correlation between the gravity-gradient noise at the interferometer's two corner test masses. It is a universal, mode-independent function of the waves' horizontal phase shift in traveling from one test mass to the other:

$$
\gamma_{J}=\gamma\left(\omega l / c_{H J}\right)
$$

Here $\omega=2 \pi f$ is the waves' angular frequency, $l \sim 5 \mathrm{~m}$ is the distance between the two corner test masses, $c_{H J}$ is the horizontal phase speed $c_{H}$ for mode $J$, and $\omega / c_{H} \equiv k$ is the mode's horizontal wave number. For frequencies and modes of interest to us, the argument $y=\omega l / c_{H J}$ of $\gamma$ is of order unity. The function $\gamma(y)$, given by

$$
\gamma(y) \equiv \sqrt{1+\frac{1}{2 \pi} \int_{0}^{2 \pi} \cos \phi \sin \phi \cos \left(y \frac{\cos \phi+\sin \phi}{\sqrt{2}}\right) d \phi}=\sqrt{1-\frac{1}{2} J_{2}(y)}
$$

[where $J_{2}(y)$ is the second Bessel function of the first kind] is plotted in Fig. 1. As Fig. 1 shows, $\gamma(y)$ is within about 10 per cent of unity for all frequencies, so we shall regard it as unity througout the rest of this manuscript, except in Appen$\operatorname{dix}$ A.

The second term, $\Gamma_{J}$, in Eq. (1.17) for $\beta_{J}$ describes the attenuation of the gravity gradient noise due to the height $\mathcal{H}$ of the test masses above the earth's surface. We show in Appendix A that

$$
\Gamma_{J}=\exp \left(-\omega \mathcal{H} / c_{H J}\right)
$$

For LIGO interferometers $\mathcal{H}$ is about $1.5 \mathrm{~m}$, the frequency of greatest concern is $f=\omega / 2 \pi \simeq 10 \mathrm{~Hz}$ (cf. Fig. 2 below), and at quiet times the dominant contribution to the noise probably comes from the RF mode (cf. Sec. I D 2) for which,

\footnotetext{
${ }^{2}$ Geophysicists use the name spectral ratio for $1 / \mathcal{A}$ $=1 /($ anisotropy ratio $)$.
}

near $10 \mathrm{~Hz}, c_{H} \simeq 330 \mathrm{~m} / \mathrm{s}$ (cf. Figs. 6 and 9); correspondingly, $\Gamma_{R F} \simeq 0.75$. For other modes, $c_{H}$ will be larger so $\Gamma_{J}$ will be closer to unity than this. For this reason, throughout the rest of this paper, except in Appendix A, we shall approximate $\Gamma_{J}$ by unity. With $\gamma_{J}$ and $\Gamma_{J}$ both approximated as unity, we henceforth shall blur the distinction between $\beta_{J}$ and $\beta_{J}^{\prime}$, treating them as equal [cf. Eq. (1.17)].

In Appendix $\mathrm{A}$ we derive expressions for the reduced transfer function $\beta_{J}^{\prime}(f)$ and the anisotropy ratio $\mathcal{A}_{J}$ in terms of properties of the eigenfunctions for mode $J$ : denote by $\xi_{H J}$ and $\xi_{V J}$ the mode's complex amplitudes at the Earth's surface $(z=0)$ for horizontal displacement and upward vertical displacement, so the mode's surface displacement eigenfunction is

$$
\vec{\xi}_{J}=\left(\xi_{H J} \hat{k}-\xi_{V J} \vec{e}_{z}\right) e^{i(\vec{k} \cdot \vec{x}-\omega t)}
$$

where $\vec{e}_{z}$ is the unit vector pointing downward and $\hat{k}=\vec{k} / k$ is the unit vector along the propagation direction. Also, denote 

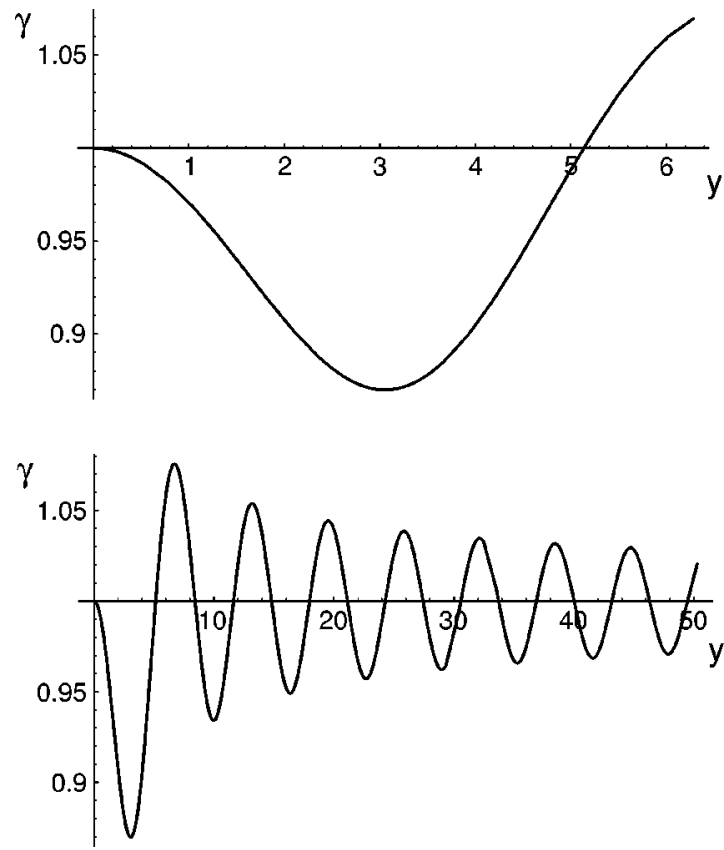

FIG. 1. The function $\gamma(y)$ that accounts for correlations of seismic gravity-gradient noise in the two corner test masses. This function is given analytically by Eq. (1.19), and it appears in all of the reduced transfer functions: $\beta_{J}(f)=\beta_{J}^{\prime}(f) \gamma\left(2 \pi f l / c_{H J}\right) \Gamma_{J}(f)$.

by $\mathcal{R}_{J}(z)$ the mode's amplitude for the fractional perturbation of density $\delta \rho / \rho$ at depth $z$ below the surface, so

$$
\frac{\delta \rho_{J}}{\rho}=\left[\xi_{V J} \delta(z)+\mathcal{R}_{J}(z)\right] e^{i(\vec{k} \cdot \vec{x}-\omega t)} .
$$

Here the term $\xi_{V J} \delta(z)$ accounts for the mass moved above $z=0$ by the upward vertical displacement $\xi_{V}$. Then, we show in Appendix A [Eq. (A7)] that

$$
\mathcal{A}_{J}=\sqrt{2} \frac{\left|\xi_{V J}\right|}{\left|\xi_{H J}\right|}
$$

where the $\sqrt{2}$ comes from the fact that when this mode is incoherently excited over all horizontal directions $\hat{k}$, its rms horizontal amplitude along any chosen direction is $\left|\xi_{H J}\right| / \sqrt{2}$. Similarly, we show in Appendix A [Eq. (A21)] that

$$
\beta_{J}^{\prime}(f)=\sqrt{\frac{3 / 2}{\left|\xi_{H J}\right|^{2}+\left|\xi_{V J}\right|^{2}}}\left|\xi_{V J}+\int_{0}^{\infty} \mathcal{R}_{J}(z) e^{-k z} d z\right|,
$$

where $k=\omega / c_{H J}$ for mode $J$. We shall refer to the $\xi_{V J}$ term in Eq. (1.24) as the surface source of gravity gradients, and the $\int \mathcal{R}{ }_{J} e^{-k z} d z$ term as the subsurface source.

Note that the influence of a given density perturbation dies out as $e^{-k z}$, so unless $\mathcal{R}_{J}(z)$ increases significantly with depth, the seismic gravity gradients arise largely from depths shallower than the gravity-gradient e-folding length

$$
\mathcal{Z}_{\mathrm{sgg}}=\frac{1}{k}=\frac{c_{H J}}{2 \pi f}=16 \mathrm{~m} \frac{\left(c_{H J} / 1000 \mathrm{~ms}^{-1}\right)}{(f / 10 \mathrm{~Hz})} .
$$

This has a simple explanation: (i) to produce much gravitational force on a test mass, a compressed bit of matter must reside at an angle $\alpha \gtrsim \pi / 4$ to the vertical as seen by the test mass, and (ii) bits of matter all at the same $\alpha \gtrsim \pi / 4$ and at fixed time have fractional compressions $\delta \rho / \rho$ that oscillate with depth $z$ as $e^{i k x}=e^{i k z \tan \alpha}$, and that therefore tend to cancel each other out below a depth $1 /(k \tan \alpha) \sim 1 / k$.

From Eq. (1.24) we can estimate the magnitude of the reduced transfer function. The mode's fractional density perturbation $\mathcal{R}_{J}$ is equal to the divergence of its displacement eigenfuction (aside from sign), which is roughly $k \xi_{H J}$ and often does not vary substantially over the shallow depths $z$ $\leqslant \mathcal{Z}_{\text {sgg }}$ where the gravity gradients originate. Correspondingly, the integral in Eq. (1.24) is $\sim \xi_{H J}$, so $\beta_{J}^{\prime}$ $\sim \sqrt{1.5\left|\xi_{H J}+\xi_{V J}\right|^{2} /\left(\left|\xi_{H J}\right|^{2}+\left|\xi_{V J}\right|^{2}\right)} \sim 1$, since the horizontal and vertical displacements are comparable.

As we shall see in Secs. II and III B 3 below, for RP modes the gravity gradients produced by the surface and subsurface sources tend to cancel (a consequence of mass conservation), so $\beta^{\prime}$ actually tends to be somewhat smaller than unity,

$$
\beta_{\mathrm{RP}}^{\prime} \lesssim 0.15,
$$

while for RF and RS modes, the surface source tends to dominate, so

$$
\beta_{\mathrm{RF}}^{\prime} \sim \beta_{\mathrm{RS}}^{\prime} \sim \frac{1}{\sqrt{2}} \sqrt{\frac{3}{1+2 \mathcal{A}_{J}^{2}}} \sim \frac{1}{\sqrt{2}}=0.7 .
$$

If we had normalized our transfer function to the vertical displacement spectrum $|\widetilde{Z}(f)|$ instead of the directionaveraged spectrum $|\widetilde{W}(f)|$ [Eq. (1.6)], then for modes in which the surface source strongly dominates, $\beta_{J}^{\prime}$ would be $1 / \sqrt{2}$ independently of the mode's anisotropy ratio.

In Secs. III and IV and associated Appendices, we shall derive, for each low-order Rayleigh mode at Hanford and Livingston, the reduced transfer function $\beta_{J}^{\prime}$ and the anisotropy ratio $\mathcal{A}_{J}$. In Sec. I D, we shall discuss the likely and the allowed weightings $w_{J}$ of the various modes [subject to the constraints (1.12) and (1.15)], and shall estimate the resulting net reduced transfer functions $\beta(f)$ for the two sites and for quiet and noisy times (Table I).

Henceforth we typically shall omit the subscript $J$ that denotes the mode name, except where it is needed for clarity.

\section{Saulson's analysis and transfer function}

In his original 1983 analysis of seismic gravity-gradient noise [8], Saulson was only seeking a first rough estimate, so he used a fairly crude model. He divided the earth near a test mass into regions with size $\lambda_{P} / 2$ (where $\lambda_{P}$ is the wavelength of a seismic P-wave), and he idealized the masses of these regions as fluctuating randomly and independently of each other due to an isotropic distribution of passing Pwaves. Saulson's final analytic result [his Eq. (21)] was the transfer function (1.6) with $\beta=1 / \sqrt{3}$.

Saulson's 1983 numerical estimates [8] of the seismic gravity-gradient noise were based on seismic noise levels 
$\widetilde{W}(f)=0.5 \times 10^{-8}(10 \mathrm{~Hz} / f)^{2} \mathrm{~cm} / \sqrt{\mathrm{Hz}}$ for " average sites" and a factor 10 lower than this for "quiet sites." The resulting gravity-gradient noise $\widetilde{x}(f)=T(f) \widetilde{W}(f)$ was substantially below the projected vibrational seismic noise in (seismically well isolated) "advanced" LIGO interferometers [1].

In updating these estimates for his recent monograph [10], Saulson noted that his original "average" and "quiet" sites were based on measurements at underground seismological stations. Surface sites, such as those chosen for LIGO and VIRGO, are far noisier than underground sites in the relevant frequency band, $3 \mathrm{~Hz} \lesssim f \leqq 30 \mathrm{~Hz}$, because of surface seismic waves. More specifically, even though the chosen LIGO sites (at Hanford, Washington and Livingston, Louisiana) are among the more quiet locations that were studied in the LIGO site survey, their noise at typical times is approximately isotropic $[\widetilde{Z}(f) \sim \widetilde{X}(f) \sim \widetilde{W}(f)]$ and has approximately the following form and magnitude $[20,21]$

$$
\begin{aligned}
\widetilde{W}(f) & =1 \times 10^{-7} \frac{\mathrm{cm}}{\sqrt{\mathrm{Hz}}} \text { at } 1<f<10 \mathrm{~Hz}, \\
& =1 \times 10^{-7} \frac{\mathrm{cm}}{\sqrt{\mathrm{Hz}}}\left(\frac{10 \mathrm{~Hz}}{f}\right)^{2} \text { at } f>10 \mathrm{~Hz} .
\end{aligned}
$$

This so-called standard LIGO seismic spectrum is 20 times larger than at Saulson's original "average" sites for $f$ $\geqslant 10 \mathrm{~Hz}$. Correspondingly, Saulson pointed out in his update, the seismic gravity-gradient noise may stick up above the vibrational seismic noise in "advanced" LIGO interferometers. ${ }^{3}$ On the other hand, at very quiet times-at night and with winds below $5 \mathrm{mph}$-the LIGO seismic ground noise $\widetilde{W}(f)$ can be as low as $\sim 1 / 10$ the level (1.27), thereby pushing Saulson's seismic gravity-gradient noise well below the vibrational seismic noise of an "advanced", LIGO interferometer.

\section{Our analysis and transfer function}

Saulson's new, more pessimistic estimates of the seismic gravity gradient noise triggered us to revisit his derivation of the transfer function $T(f)$ from seismic ground motions to detector noise. Our analysis consists of:

(i) splitting the ambient seismic motions into Love and Rayleigh modes (body of this paper and Appendices);

(ii) computing the reduced transfer function for each mode and for models of the geological strata at each LIGO site (body and Appendices);

\footnotetext{
${ }^{3}$ Saulson informs us that in evaluating the noise at the LIGO sites, he made an error of $\sqrt{3}$; his transfer function and the standard LIGO seismic spectrum actually predict a noise level $\sqrt{3}$ smaller than he shows in Fig. 8.7 of his book [10]. When this is corrected, his predicted noise, like ours, is below the "advanced" LIGO noise curve, though only slightly so near $10 \mathrm{~Hz}$.
}

TABLE I. Reduced transfer functions $\beta^{\prime}$ predicted for Hanford and Livingston by our 4-layer models; and $\beta_{L}^{\prime}$, the value of $\beta^{\prime}$ when enough Love waves are added to bring the anisotropy ratio down to the quiet-time values observed at the two sites $(\mathcal{A} \simeq 1$ for Hanford, $\mathcal{A} \simeq 0.6$ for Livingston).

\begin{tabular}{llccc}
\hline \hline Modes & $\begin{array}{c}\text { Hanford } \\
\beta^{\prime}\end{array}$ & $\begin{array}{c}\text { Hanford } \\
\beta_{L}^{\prime}\end{array}$ & $\begin{array}{c}\text { Livingston } \\
\beta^{\prime}\end{array}$ & $\begin{array}{c}\text { Livingston } \\
\beta_{L}^{\prime}\end{array}$ \\
\hline RF $f<10 \mathrm{~Hz}$ & $0.4-0.85$ & $0.35-0.6$ & $0.65-0.9$ & $0.35-0.45$ \\
RF $f>10 \mathrm{~Hz}$ & 0.85 & 0.6 & $0.65-0.9$ & $0.35-0.45$ \\
RS & $0.4-1.4$ & $0.4-1.05$ & $0-1.2$ & $0-0.9$ \\
RP & $0-0.15$ & $0-0.15$ & $0.02-0.13$ & $0.01-0.06$ \\
\hline \hline
\end{tabular}

(iii) using seismic measurements at the LIGO sites and geophysical lore based on other sites to estimate the mode mixture present at the two sites under both quiet and noisy conditions (this section); and

(iv) evaluating for these mode mixtures the expected reduced transfer function and resulting noise (this section).

\section{Our reduced transfer functions}

Table I summarizes the results of our model computations for each LIGO site. Shown there are the range of computed reduced transfer functions $\beta^{\prime}$ for specific types of Rayleigh modes, and the range of net reduced transfer functions $\beta_{L}^{\prime}$ that would result if each Rayleigh mode were mixed with enough Love waves to bring its (often rather high) anisotropy ratio $\mathcal{A}$ down to the level typical of quiet times at the LIGO sites $(\mathcal{A} \simeq 1.0$ at Hanford [21], $\mathcal{A} \simeq 0.6$ at Livingston [20]).

The modes shown in Table I are the RF mode, the RS modes with no sign of RP admixture, and the RP modes. The $\mathrm{RF}$ and RS modes usually have $\beta^{\prime}$ in the range 0.4 to 1.2 , though in special cases it can sink toward zero. By contrast, the RP modes always have small $\beta^{\prime}: 0$ to 0.15 . This marked difference arises from the fact that for RF and RS the (largely S-wave) surface source tends to dominate over the (entirely P-wave) subsurface source; while for RP, mass conservation guarantees that the two sources (both largely Pwave) will be nearly equal, but opposite in sign, and will nearly cancel. (If the surface source were absent, the pattern would be reversed: the subsurface source $\int \mathcal{R}$, which arises from compressional density perturbations, tends to be weak for RS modes because they consist primarily of noncompressional S-waves, but is strong for RP modes since they consist primarily of compressional P-waves; so $\beta^{\prime}$ would be small for RS and large for RP.)

\section{Modes actually present and resulting seismic noise}

There is little direct evidence regarding which modes contribute to the ambient surface motions and thence to the gravity-gradient noise at the LIGO sites during quiet times. Past seismic measurements do not shed much light on this issue. In the concluding section of this paper (Sec. V), we shall propose measurements that could do so.

Fortunately, the nature of the ambiently excited modes has been studied at other, geophysically similar sites (hori- 
zontally stratified alluvia over bedrock). The preponderance of evidence suggests that at quiet times the surface motions at such sites and in our frequency band are due to a mixture of Love waves and the fundamental Rayleigh mode RF plus perhaps a few low order RS modes [22-26]. In at least one case, some amount of RP excitation is also seen [27]; these RP excitations are ascribed to "cultural noise" (noise generated by human activity of some sort) near the measurement site. Deep borehole measurements indicate that RP dominates at very great depths $(\sim 5 \mathrm{~km})[28]$; this is probably not relevant to our analysis, however. It merely indicates that very deep down, the majority of the surface waves have damped away, leaving only some residual RP modes. The deep motions are typically an order of magnitude or two smaller than the surface motions; cf. Sec. I C.

On this basis, we presume that at quiet times the net reduced transfer function is about that for the RF mode, with enough admixed Love waves to bring the net $\mathcal{A}$ down to the typical quiet-time values of 1.0 for Hanford and 0.6 for Livingston. In other words, $\beta_{\text {net }}^{\prime}$ is about equal to $\beta_{L}^{\prime}$ for the RF mode:

$$
\begin{aligned}
\beta_{\text {net, quiet times }}^{\prime} & \simeq 0.35-0.45 \text { at Livingston, } \\
& \simeq 0.35-0.6 \text { at Hanford }
\end{aligned}
$$

We have folded these quiet-time estimates for $\beta^{\prime}$ into the standard LIGO seismic spectrum (1.27) to obtain the gravitygradient noise estimates shown as the dark gray band in Fig. 2. The thickness of the band indicates the range of our $\beta^{\prime}$ [Eq. (1.28)]: 0.35 to 0.6. To produce this plot, we took expression (1.8) for the transfer function $T(f)$ with $\gamma$ and $\Gamma$ set to unity, so $\beta=\beta^{\prime}$ [cf. Eq. (1.17)]. Then, we multiplied this by the standard LIGO seismic spectrum (1.27) for the ground displacement with an assumed density $\rho=1.8 \mathrm{~g} / \mathrm{cm}^{3}$. This yields

$$
\begin{aligned}
\tilde{h}_{\mathrm{SGG}}(f)= & \frac{\beta^{\prime}}{0.6} \frac{6 \times 10^{-23}}{\sqrt{\mathrm{Hz}}}\left(\frac{10 \mathrm{~Hz}}{f}\right)^{2}, \\
& 3 \mathrm{~Hz} \lesssim f<10 \mathrm{~Hz}, \\
= & \frac{\beta^{\prime}}{0.6} \frac{6 \times 10^{-23}}{\sqrt{\mathrm{Hz}}}\left(\frac{10 \mathrm{~Hz}}{f}\right)^{4}, \\
& 10 \mathrm{~Hz}<f \leqslant 30 \mathrm{~Hz},
\end{aligned}
$$

which we plotted for the indicated values of $\beta^{\prime}$.

At very quiet times, the ambient seismic spectrum near 10 $\mathrm{Hz}$ can be as much as a factor $\sim 10$ lower than the standard LIGO spectrum assumed in Eq. (1.29) and Fig. 2, and correspondingly the quiet-time gravity gradient noise can be a factor $\sim 10$ lower.

At noisier times, there appear to be excitations of a variety of RF, RS and RP modes. For example, at the LIGO sites, time delays in correlations between surface motions at the corner and the end stations reveal horizontal propagation speeds $c_{H} \sim 5000 \mathrm{~m} / \mathrm{s}$, corresponding to deeply seated RPmodes (although for the most part these modes are seen at frequencies too low to be of interest in this analysis- $f$

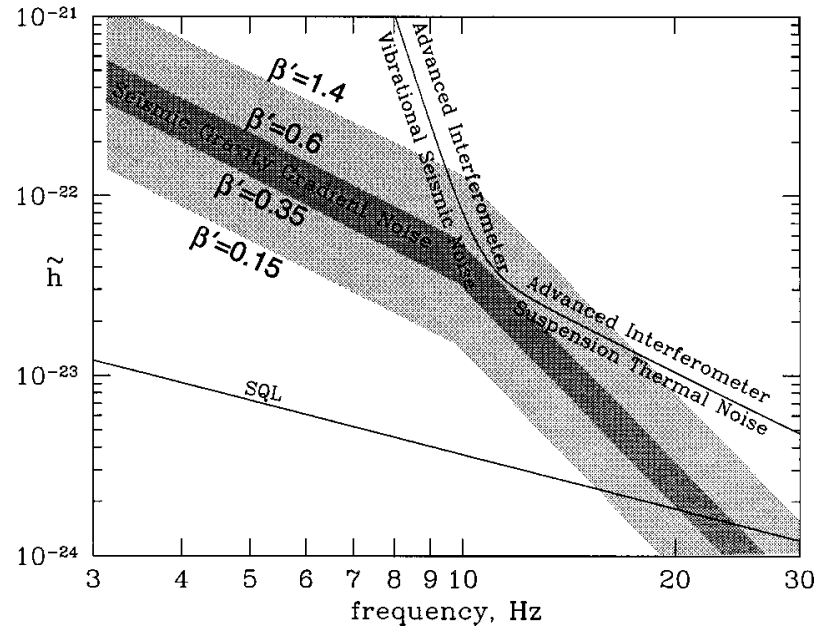

FIG. 2. Seismic gravity-gradient noise in a LIGO interferometer. In this figure, we assume that the direction-averaged spectrum of earth displacements has the form of the standard LIGO seismic spectrum, Eq. (1.27). The edges of the gray bands are for the indicated values of the reduced transfer function $\beta^{\prime}$ (assumed equal to $\beta$; i.e., for $\gamma$ and $\Gamma$ approximated as unity). The dark gray band is our estimate of the range of noise for quiet times. The gray bands, both light and dark, are for noisy times, assuming the standard LIGO seismic spectrum (1.27). At very quiet times, the ground spectrum can be a factor $\sim 10$ smaller than (1.27), which will lower these bands accordingly. Conversely, at noisy times the ground spectrum can be larger, raising these bands. Also shown for comparison is the projected noise in an "advanced" LIGO interferometer, and the standard quantum limit (SQL) for an interferometer with one tonne test masses. The SQL is the square root of Eq. (122) of Ref. [31]. The "advanced"' interferometer noise is taken from Fig. 7 of Ref. [1], with correction of a factor 3 error in the suspension thermal segment (Fig. 7 of Ref. [1] is a factor 3 too small, but Fig. 10 of that reference is correct, for the parameters listed at the end of the section "LIGO Interferometers and Their Noise").

$\lesssim 0.2 \mathrm{~Hz}[29,30])$. Moreover, the measured anisotropy ratios can fluctuate wildly from $\sim 0.2$ to $\sim 5$ at noisy times, suggesting a wildly fluctuating mixture of RF, RS, RP, and Love modes. Scrutinizing not only Table I but also the range of $\beta^{\prime}$ shown in Figs. 7, 8, 10 and 11 which underlie that table, and keeping in mind that Love modes with vanishing $\beta^{\prime}$ will also be present, we estimate that the fluctuations of $\beta^{\prime}$ at noisy times will be confined to the range

$$
\beta_{\text {net, noisy times }}^{\prime} \simeq 0.15-1.4 \text {. }
$$

We have folded this estimate into the standard LIGO seismic spectrum to obtain the upper and lower edges of the light gray band in Fig. 2. The gray bands, light and gray taken together, are our best estimate of the range of seismic gravity-gradient noise at noisy times, assuming the standard LIGO seismic spectrum. Since, at noisy times, the seismic spectrum can be somewhat higher than the standard one, the gravity-gradient noise will be correspondingly higher.

For the next few years, the most important application of these estimates is as a guide for the development of seismic isolation systems and suspension systems for LIGO. There is 
not much point in pushing such systems so hard that the vibrational seismic or the suspension thermal noise is driven far below our lowest estimates of the seismic gravitygradient noise [bottom of the black line in Fig. 2, lowered by the amount that the actual very quiet time spectrum falls below the standard LIGO spectrum (1.27)] - unless corresponding steps are taken to mitigate the seismic gravity gradient noise; see Sec. V.

In Fig. 2 we compare our predicted seismic gravity gradient noise to the projected noise in "advanced" LIGO interferometers and to the standard quantum limit for an interferometer with one tonne test masses ("SQL"). Notice that our lower bound on the seismic gravity-gradient noise is everywhere smaller than the "advanced" interferometer noise, but it is larger than the SQL at frequencies below $\sim 20 \mathrm{~Hz}$. Our lower bound rises large enough below $\sim 10 \mathrm{~Hz}$ to place limits on seismic-isolation and suspension-noise R\&D that one might contemplate doing at such frequencies.

The remainder of this paper is organized as follows: we begin in Sec. II by discussing Rayleigh waves and seismic gravity-gradient noise in the idealized case of a homogeneous half space (not a bad idealization for some regions of some modes at Hanford and Livingston). Then we develop multilayer geophysical models for Hanford and Livingston and use them to derive the reduced transfer functions for the various Rayleigh modes (Secs. III and IV). We conclude in Sec. V with a discussion of the uncertainties in our analysis and research that could be undertaken to reduce the uncertainties, and also a discussion of the physical interaction of the seismic waves with the foundations of the LIGO facilities, and of ways to somewhat reduce the gravity gradient noise if it ever becomes a serious problem in LIGO interferometers. Mathematical details of our analysis are confined to Appendices. Those Appendices may form a useful foundation for analyses of seismic gravity-gradient noise at other sites.

\section{HOMOGENEOUS HALF SPACE}

\section{A. Fundamental Rayleigh mode}

As a first rough guide to seismic gravity-gradient noise, we idealize the LIGO sites as a homogeneous half space with density $\rho$, Poisson ratio $\nu, \mathrm{S}$-wave speed $c_{S}$ and $\mathrm{P}$-wave speed $c_{P}$ given by

$$
\begin{gathered}
\rho=1.8 \mathrm{~g} / \mathrm{cm}^{3}, \quad \nu=0.33, \quad c_{P}=440 \mathrm{~m} / \mathrm{s}, \\
c_{S}=220 \mathrm{~m} / \mathrm{s} .
\end{gathered}
$$

(These are the measured parameters of the surface material at Livingston; for Hanford, the parameters are only a little different; cf. Sec. III A below.)

This homogeneous half space can only support the RF mode, as mentioned in the Introduction. The theory of the RF mode and the seismic gravity-gradient noise that it produces is sketched in Appendix B. Here we summarize the results.
The RF mode propagates with a horizontal speed $c_{H}$ that depends solely on the Poisson ratio. It is a bit slower than the speed of S-waves, and is much slower than P-waves. For the above parameters,

$$
c_{H}=0.93 c_{S}=205 \mathrm{~m} / \mathrm{s} ;
$$

cf. Eq. (B1). Correspondingly, the waves' horizontal wave number $k$ and horizontal reduced wavelength are

$$
\frac{\lambda}{2 \pi}=\frac{1}{k}=3.3 \mathrm{~m}\left(\frac{10 \mathrm{~Hz}}{f}\right) \text {. }
$$

Because $c_{H}<c_{S}<c_{P}$, RF waves are evanescent vertically: the P-waves die out with depth $z$ as $e^{-q k z}$, and the $\mathrm{SV}$-waves as $e^{-s k z}$, where

$$
\begin{aligned}
& q=\sqrt{1-\left(c_{H} / c_{P}\right)^{2}}=0.88, \\
& s=\sqrt{1-\left(c_{H} / c_{S}\right)^{2}}=0.36 .
\end{aligned}
$$

Thus, the vertical $e$-folding lengths for compression (which produces seismic gravity gradients) and shear (which does not) are

$$
\begin{aligned}
& \mathcal{Z}_{P}=\frac{1}{q k}=3.7 \mathrm{~m}\left(\frac{10 \mathrm{~Hz}}{f}\right), \\
& \mathcal{Z}_{S}=\frac{1}{s k}=9.0 \mathrm{~m}\left(\frac{10 \mathrm{~Hz}}{f}\right) .
\end{aligned}
$$

These RF waves produce substantially larger vertical motions than horizontal at the Earth's surface. For waves that are horizontally isotropic, the anisotropy ratio is

$$
\mathcal{A}=\sqrt{2} \frac{q\left(1-s^{2}\right)}{1+s^{2}-2 q s}=2.2 .
$$

This large ratio is indicative of the fact that RF waves contain a large component of P-waves. As mentioned in the Introduction, this is substantially larger than the values typically observed at the LIGO sites in the band $3 \mathrm{~Hz} \lesssim f$ $\leqslant 30 \mathrm{~Hz}$-seismic measurements taken at those sites [20,21] show that, at quiet times, $\mathcal{A} \simeq 1.0$ at Hanford, $\mathcal{A} \simeq 0.6$ at Livingston. Thus, RF waves cannot alone be responsible for the seismic motions. To the extent that our homogeneous-halfspace model is realistic, RF waves must be augmented by a large amount of horizontally-polarized S-waves ("SHwaves"), which have $\mathcal{A}=0$.

$\mathrm{RF}$ waves produce a reduced transfer function

$$
\beta^{\prime}=\sqrt{\frac{3\left(1+s^{2}-2 q\right)^{2}}{2\left(1+s^{2}\right)\left[\left(1+s^{2}\right)\left(1+q^{2}\right)-4 q s\right]}}=0.86 .
$$

This $\beta^{\prime}$ is produced primarily by the surface source $\xi_{V}$ in Eq. (1.24); if there were no surface source, the subsurface term $\int \mathcal{R}$ (arising solely from the $\mathrm{P}$-wave compressions) would produce the far smaller value $\beta^{\prime}=0.17$. When the RF waves are augmented by enough Love waves to reduce the net $\mathcal{A}$ to 1.0 (Hanford) or 0.6 (Livingston), they produce a net reduced transfer function [Eq. (1.16) with primes added to the $\beta$ 's] 


$$
\beta_{L}^{\prime}=0.59 \text { (Hanford), } 0.40 \text { (Livingston). }
$$

As we shall see in the next two sections, the Earth is strongly stratified over the relevant vertical length scales at both Hanford and Livingston, and this gives rise to significant differences from the homogeneous-half-space model. Nevertheless, as discussed in the Introduction (Sec. I D 2), it is likely that at quiet times the RF mode produces the dominant gravity-gradient noise. Stratification modifies this RF mode somewhat from the description given here; however, as we shall see (Figs. 7 and 10), these modifications typically alter its anisotropy ratio and reduced transfer function by only a few tens of percent. Thus, the homogeneous-halfspace model may be a reasonable indicator of seismic gravity-gradient noise in LIGO at quiet times.

\section{B. P-up and SV-up waves}

The principal effect of stratification is to produce a rich variety of normal-mode oscillations, in which mixtures of $\mathrm{SV}$ - and P-waves resonate in leaky cavities formed by the strata. These oscillations are Rayleigh-mode overtones, whose (rather complex) theory is sketched in Appendices $\mathrm{C}$ and D and discussed in Secs. III and IV. In this subsection we will momentarily ignore that fact, and will seek insight from a much simpler analysis that gives results which agree approximately, and in some cases quite well, with those of the Rayleigh-overtone theory.

If the top layer (labeled by a subscript 1) has a thickness $D_{1}$ larger than half a vertical wavelength of the waves' oscillations, $D_{1}>\left(c_{P 1} / 2 f\right) / \sqrt{1-\left(c_{P 1} / c_{H}\right)^{2}}$ for P-waves and similarly for S-waves [cf. Eq. (3.2) below and associated discussion], then the trapped modes can be thought of as propagating upward through the top layer, reflecting at the earth's surface, and then propagating back downward. By ignoring the effects of the interfaces below, these waves can be idealized as traveling in a homogeneous half space.

The behavior of these waves depends on the mixture of Pand $\mathrm{SV}$-waves that composes them as they propagate upward. Because these two components will superpose linearly, we can decompose the mixture and treat the P-wave parts and SV-wave parts separately. We will call these components P-up and SV-up waves. In Appendix E, we derive simple analytic formulas for the anisotropy ratio $\mathcal{A}$ and reduced transfer function $\beta^{\prime}$ for P-up and SV-up waves, and in Figs. 3 and 4 we graph those formulas. In these plots, for concreteness, we have chosen $c_{S}=c_{P} / 2$.

Consider, first, the P-up waves (solid curves in Figs. 3 and 4). Due to Snell's law [cf. Eq. (3.1) below], these waves propagate at an angle $\alpha_{P}=\arcsin \left(c_{P} / c_{H}\right)$ to the vertical. Such propagating waves can therefore exist only for $c_{H}>c_{P}$; when $c_{H}<c_{P}$, P-waves are evanescent. For this reason, in the figures we plot on the abscissa the ratio $c_{P} / c_{H}$ running from 0 to 1 . When P-up waves hit the surface, some of their energy is converted into $\mathrm{SV}$-waves propagating downward at an angle $\alpha_{S}=\arcsin \left(c_{S} / c_{H}\right)$; the rest of the energy goes into reflected $\mathrm{P}$-waves. The resulting combination of upgoing $\mathrm{P}$ and downgoing $\mathrm{P}$ - and $\mathrm{SV}$-waves gives rise to the anisotropy and reduced transfer functions shown in the figures.

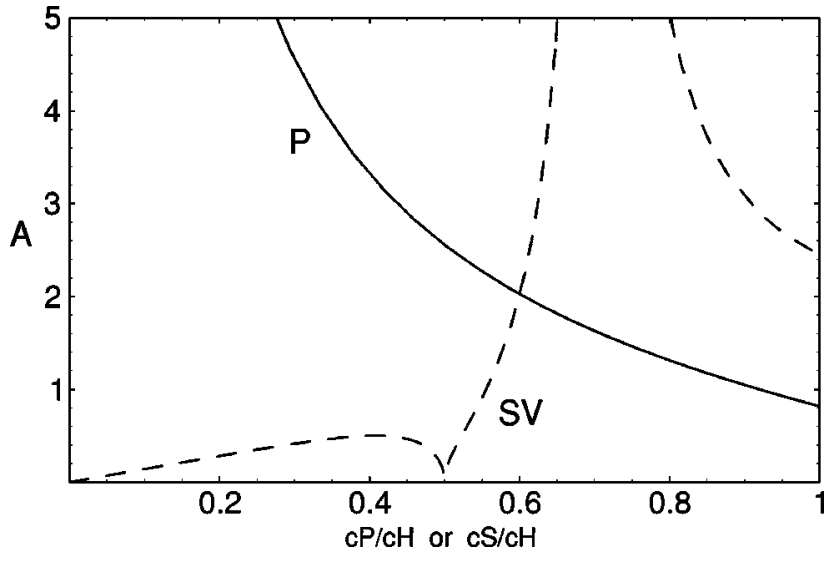

FIG. 3. Anisotropy ratio for waves that propagate upward in a homogeneous half space, reflect off the Earth's surface, and propagate back downward. The curve " $\mathrm{P}$ '" is for the case when the upward propagating waves are pure $\mathrm{P}$ ( $\mathrm{P}$-up waves), in which case the abscissa is $c_{P} / c_{H} \equiv \sin \alpha_{P}$; " $\mathrm{SV}$ ' is for SV-up waves, with abscissa $c_{S} / c_{H} \equiv \sin \alpha_{S}$. It is assumed that $c_{P}=2 c_{S}$; this is approximately the case for the surface layers at Hanford and Livingston.

For $c_{H} \gg c_{P}$ the waves travel nearly vertically. Their Pcomponents produce vertical motions, while the much weaker SV-waves created on reflection produce horizontal motions. As a result, $\mathcal{A}$ is large, diverging in the limit $c_{H}$ $\rightarrow \infty$, and decreasing gradually to near unity as $c_{H} \rightarrow c_{P}$. As we shall see below, this is typical: when $\mathrm{P}$-waves predominate in a wave mixture (RP modes), $\mathcal{A}$ is typically somewhat larger than unity.

For these P-up waves, the gravity gradients produced by the surface source cancel those from the subsurface source in the limit $c_{H} \gg c_{P}$, causing $\beta^{\prime}$ to vanish. As $c_{H}$ is reduced (moving rightward in Fig. 4), the cancellation becomes imperfect and $\beta^{\prime}$ grows, though never to as large a value as $\beta^{\prime}$ would have in the absence of the surface term $(\sim 1.3-2.4)$. The surface-subsurface cancellation is easily understood. In the limit $c_{H} \gg c_{P}$, the P-waves propagate nearly vertically, with vertical reduced wavelength for their density oscillations, $1 / k_{V}=c_{P} / \omega$, that is small compared to the gravity-gradient $e$-folding length $\mathcal{Z}_{\mathrm{sgg}}=1 / k=c_{H} / \omega$, over

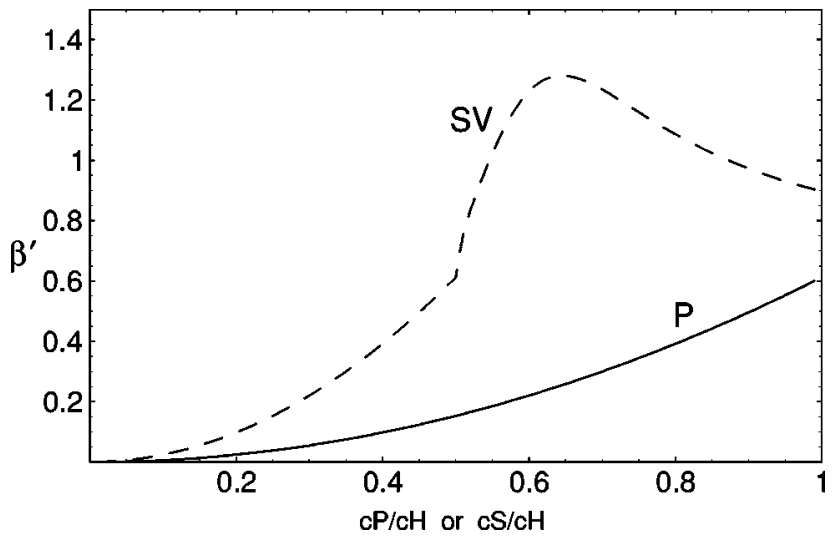

FIG. 4. Reduced transfer function for P-up and SV-up waves in a homogeneous half space with $c_{P}=2 c_{S}$. Notation is as in Fig. 3. 
which the waves' sources are integrated in Eq. (1.24) to produce the gravitational force. Therefore, the gravity gradients come from many vertical wavelengths, with adjacent ones weighted nearly equally. Because of mass conservation, the surface source plus the top quarter wavelength of subsurface source (multiplied by $\rho$ ) constitute the mass per unit area that has been raised above a node of the mode's displacement eigenfunction; and correspondingly their sum vanishes. Below that node, alternate half wavelengths of the subsurface source cancel each other in a manner that gets weighted exponentially with depth, $e^{-k z}$; their cancellation is excellent in the limit $1 / k_{V} \ll 1 / k$, i.e., $c_{H} \gg c_{P}$.

Turn now to the SV-up waves. Upon reflection from the surface, these produce a mixture of downgoing SV- and Pwaves. This mixture gives rise to the anisotropy and reduced transfer functions shown dashed in Figs. 3 and 4. Again by Snell's law, SV-up waves propagate at an angle $\alpha_{S}$ $=\arcsin \left(c_{S} / c_{H}\right)$ to the vertical; thus, propagation is possible only for $c_{H}>c_{S}$, and so we plot on the abscissa $c_{S} / c_{H}$ running from 0 to 1 . When $c_{H}>2 c_{S}=c_{P}$ (left half of graphs), the downgoing $\mathrm{P}$-waves generated at the surface can propagate; when $c_{H}<2 c_{S}=c_{P}$ (right half of graphs), the downgoing P-waves have imaginary propagation angle $\alpha_{P}$ and thus are evanescent (decay exponentially with depth). This is analogous to the phenomenon of total internal reflection which one encounters in elementary optics. The downgoing P-waves are the sole subsurface source of gravity-gradient noise, and since they are only a modest component of the SV-Up mode, the subsurface source is small. The SV-waves produce no subsurface source (no compressions), but they produce a large surface source (large surface vertical motions). This surface source is the dominant cause of the gravity-gradient noise and predominantly responsible for the rather large reduced transfer function shown in Fig. 4. Note that the maximum value, $\beta^{\prime} \simeq 1.4$, is the same as the largest $\beta^{\prime}$ for RS modes in our 4-layer models of the LIGO sites (Table I).

When propagating more or less vertically $\left(c_{H}>2 c_{S}\right)$, these SV-up waves produce small anisotropies $(\mathcal{A}$ $<0.4-$ large horizontal motions and small vertical motions). When they propagate more or less horizontally, $\mathcal{A}$ is large. The divergence of $\mathcal{A}$ at $c_{S} / c_{H}=1 / \sqrt{2}=0.707\left(\alpha_{S}=\pi / 4\right)$ occurs because the SV-up waves at this angle generate no Pwaves upon reflection; they only generate downgoing SVwaves, and the combination of the equal-amplitude up and down SV-waves produces purely vertical motions at the Earth's surface. At frequencies $f \gtrsim 20 \mathrm{~Hz}$, mode RS1 at Hanford can be approximated as an SV-up mode and exhibits this behavior; cf. Sec. III B 3 .

\section{HANFORD}

\section{A. Hanford geophysical structure}

At the LIGO site near Hanford, Washington, the top 220 $\mathrm{m}$ consists of a variety of alluvial layers (fluvial and glaciofluvial deposits of the Pliocene, Pleistocene, and Holocene eras; coarse sands and gravels, fine sands, silts, and clays, in a variety of orders). The upper $40 \mathrm{~m}$ are dry; below about 40 $\mathrm{m}$ the alluvium is water-saturated. From the base of the al-
TABLE II. Velocity profiles at the Hanford LIGO site, as extracted from Table 2.5-3, Fig. 2.5-10, and Sec. 2.5.2.5 of the Skagit Report [33]. These velocities are based on (i) cross-hole measurements (waves excited in one borehole and measured in another) down to $60 \mathrm{~m}$ depth; (ii) downhole measurements (waves excited at surface and arrivals measured in boreholes) from $z=60 \mathrm{~m}$ to $z$ $=175 \mathrm{~m}$; (iii) extrapolations of downhole measurements at other nearby locations, and surface refraction measurements (waves excited at surface and measured at surface) at the LIGO site, from $z$ $=175 \mathrm{~m}$ down into the basalt at $z>220 \mathrm{~m}$. The downhole measurements at one well (Rattlesnake Hills No. 1) have gone into the basalt to a depth of $3230 \mathrm{~m}$. Depths are in meters, velocities are in $\mathrm{m} / \mathrm{s}$.

\begin{tabular}{|c|c|c|c|}
\hline Depths & $c_{P}$ & $c_{S}$ & $\nu$ \\
\hline $0-12$ & 520 & 270 & 0.32 \\
\hline $12-24$ & 820 & 460 & 0.27 \\
\hline $24-32$ & 1000 & 520 & 0.31 \\
\hline $32-40$ & 1260 & 530 & 0.39 \\
\hline $40-50$ & 1980 & 560 & 0.46 \\
\hline $50-80$ & 2700 & 760 & 0.46 \\
\hline $80-110$ & 2700 & 910 & 0.44 \\
\hline $110-160$ & 1800 & 610 & 0.44 \\
\hline $160-210$ & 2400 & 910 & 0.42 \\
\hline $210-220$ & 2900 & 1200 & 0.40 \\
\hline $220-250$ & 4900 & 2700 & 0.28 \\
\hline $250-3230$ & $\begin{array}{r}5000-5700 \text { competent } \\
\text { basalt flows } \\
4000-5500 \text { interbeds }\end{array}$ & & \\
\hline
\end{tabular}

luvium $(220 \mathrm{~m})$ to a depth of $\sim 4 \mathrm{~km}$ lies a sequence of Columbia River basalts, and below that, bedrock $[32,33]$.

The density of the alluvial material is $\rho \simeq 1.8 \mathrm{~g} / \mathrm{cm}^{3}$, independent of layer. Velocity profiles $\left(c_{P}\right.$ and $c_{S}$ as functions of depth $z$ ) have been measured at the site by contractors in connection with two projects: LIGO [32] and the Skagit nuclear power plant [33] (which was never constructed). We have relied primarily on the Skagit report because it contains more detailed information over the range of depths of concern to us, and because there is a serious discrepancy between the two reports in the depth range 5-25 m, which contributes significantly to the seismic gravity gradients. The Skagit velocities there are more plausible than the LIGO ones. ${ }^{4}$

Table II shows velocity profiles as extracted from the Skagit report. Notice the overall gradual increase in both wave speeds. This is due to compression of the alluvia by the

\footnotetext{
${ }^{4}$ The report prepared for LIGO [32] claims $c_{P}=1400 \mathrm{~m} / \mathrm{s}, c_{S}$ $=370 \mathrm{~m} / \mathrm{s}$, corresponding to a Poisson ratio of $\nu=0.46$. This could be appropriate for water-saturated materials at this depth, but is not appropriate for the dry materials that actually lie there. The Skagit report [33] shows two layers in this range of depths: one with $c_{P}$ $=520 \mathrm{~m} / \mathrm{s}, c_{S}=270 \mathrm{~m} / \mathrm{s}$, for which $\nu=0.32$; the other with $c_{P}$ $=820 \mathrm{~m} / \mathrm{s}, c_{S}=460 \mathrm{~m} / \mathrm{s}$, for which $\nu=0.27$. For dry alluvia, these values are much more reasonable than $\nu=0.46$. We thank Alan Rohay for bringing this point to our attention.
} 
TABLE III. Four-layer model for the velocity profiles at the Hanford LIGO site. Notation: $n$-layer number, $D_{n}$-layer thickness, $c_{P n}-\mathrm{P}$-wave speed in this layer, $c_{S n}-\mathrm{S}$-wave speed in this layer, $\nu_{n}$-Poisson ratio in this layer. Depths and thicknesses are in meters, speeds are in $\mathrm{m} / \mathrm{s}$.

\begin{tabular}{llrrrc}
\hline \hline$n$ & \multicolumn{1}{c}{ Depths } & \multicolumn{1}{c}{$D_{n}$} & \multicolumn{1}{c}{$c_{P n}$} & \multicolumn{1}{c}{$c_{S n}$} & \multicolumn{1}{c}{$\nu_{n}$} \\
\hline 1 & $0-12$ & 12 & 520 & 270 & 0.32 \\
2 & $12-40$ & 28 & 900 & 500 & 0.28 \\
3 & $40-220$ & 180 & 2400 & 700 & 0.45 \\
4 & $220-4000$ & 3780 & 4900 & 2700 & 0.28 \\
\hline \hline
\end{tabular}

weight of overlying material, with a consequent increase in the areas of the contact surfaces between adjacent particles (silt, sand, or gravel) [18]. Notice also the sudden increase of $c_{P}$ and $\nu$ at $40 \mathrm{~m}$ depth, due to a transition from dry alluvia to water-saturation; the water contributes to the bulk modulus but not the shear modulus, and thence to $c_{P}$ but not $c_{S}$. Notice, finally, the large jump in both $c_{P}$ and $c_{S}$ at the $220 \mathrm{~m}$ deep transition from alluvial deposits to basalt.

We have been warned by geophysicist and seismic engineer colleagues that we should not place great faith in all the details of measured velocity profiles such as this one; and the discrepancies between the Skagit and LIGO velocity-profile measurements have reinforced this caution. As a result, from computations based on these velocity profiles (and similar profiles at Livingston), we can only expect to learn (i) the general nature of the modes to be expected at each LIGO site, (ii) how those modes' characteristics are influenced by the velocity profiles, (iii) the range of anisotropy ratios $\mathcal{A}$ and reduced transfer functions $\beta^{\prime}$ to be expected at each site, and (iv) how $\mathcal{A}$ and $\beta^{\prime}$ depend on the velocity profiles and the modes' characteristics. We cannot expect the computed, mode-by-mode details of $\mathcal{A}(f)$ and $\beta^{\prime}(f)$ to be accurateexcept, perhaps, for the shallowly seated RF mode. Nevertheless, the insights that we do gain from such computations should be of considerable help in future studies of seismic gravity-gradient noise and future attempts (if any) to mitigate it.

In this spirit, we have simplified our calculations by approximating the measured Hanford velocity profiles (Table II) with their twelve distinct layers by the simpler four-layer model shown in Table III. Layers 1 and 2 are dry alluvia, layer 3 is water-saturated alluvium, and layer 4 is basalt.

\section{B. Hanford model results}

The horizontally stratified geologies at Hanford and Livingston support a variety of Love and Rayleigh modes. (For the general character of Love and Rayleigh modes see, e.g., Refs. $[15,16]$ and the brief discussion in the introduction of this paper.) We shall focus on Rayleigh modes in this section, since they are the sole producers of seismic gravitygradient noise.

In each geological layer, consider a specific Rayleigh mode. It consists of a superposition of plane-fronted P- and $\mathrm{SV}$-waves. Because each layer is idealized as homogeneous, the mode's SV- and P-waves are decoupled within the layer.
However, they are coupled at layer interfaces and the Earth's surface by the requirement that material displacement and normal stress be continuous across the interface (or with the atmosphere in the case of the Earth's surface). The details of this coupling and its consequences are worked out in Appendix C.

In each layer, the mode's P- and SV-components propagate at different angles to the vertical: $\alpha_{P n}$ for the P-waves in layer $n$ and $\alpha_{S n}$ for the SV-waves. However, the components must all move with the same horizontal speed

$$
c_{H}=\frac{\omega}{k}=\frac{c_{P n}}{\sin \alpha_{P n}}=\frac{c_{S n}}{\sin \alpha_{S n}}
$$

(Snell's law), and they must all have the same horizontal wave number $k$ and frequency $\omega=2 \pi f$.

Each mode can be characterized by its dispersion relation for horizontal motion $\omega(k)$, or equivalently $c_{H}(f)$. It will be helpful, in sorting out the properties of the modes, to understand first what their dispersion relations $c_{H}(f)$ would be if their SV-wave components were decoupled from their P-wave components. We shall do so in the next subsection, and then examine the effects of coupling in the following subsection. Note that we shall ignore the effects of damping in these two subsections, since the lengthscales involved are less than (or at most of the same order as) the dissipation lengthscales of both P- and SV-waves [cf. Eq. (1.5)].

\section{P-SV decoupling approximation}

Recall that we denote by RP $n$ the $n$th Rayleigh mode of P-type and by RS $n$ the $n$th Rayleigh mode of SV-type. In the approximation of P-SV decoupling, Mode RP $n$ with horizontal speed $c_{H}$ propagates from the earth's surface through sequences of strata (generating no SV-waves) until it reaches a depth $D_{P}$ where $c_{P}$ first exceeds $c_{H}$. At that location, it reflects and returns to the surface, and then is reflected back downward. The mode's dispersion relation $c_{H}(f)$ is determined by the resonance condition that the reflected waves arrive at the surface in phase with the original downgoing waves.

This resonance condition is evaluated most easily by following the (locally) planar waves vertically downward to their reflection point (the location $z=D_{P}$ where $c_{P}$ first reaches $c_{H}$ ) and then back up, thereby returning precisely to the starting point. On this path, the vertical component of the wave vector is

$$
k_{V}=\frac{\omega}{c_{P}} \cos \alpha_{P}=\frac{\omega}{c_{P}} \sqrt{1-\left(c_{P} / c_{H}\right)^{2}}=k \cot \alpha_{P},
$$

where we have used Snell's law (3.1) to infer $\cos \alpha_{P}$ $=\sqrt{1-\left(c_{P} / c_{H}\right)^{2}}$. The waves' corresponding waves' total round-trip phase shift is

$$
\Delta \Phi=2 \int_{0}^{D_{P}} \frac{\omega}{c_{P}} \sqrt{1-\left(c_{P} / c_{H}\right)^{2}} d z+\delta \Phi_{\text {interfaces }} .
$$

Here $\delta \Phi_{\text {interfaces }}$ is the total phase shift acquired at the interfaces between strata and upon reflecting at the Earth's sur- 

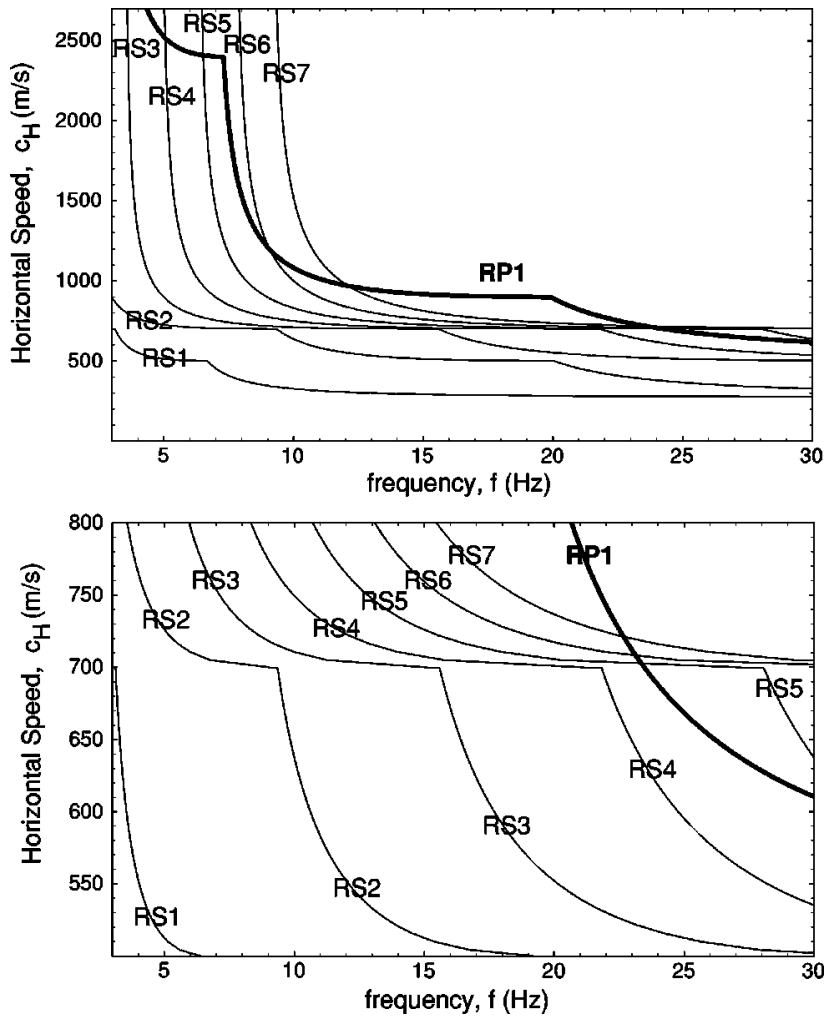

FIG. 5. Dispersion relations for the 4-layer Hanford model, as computed using the P-SV decoupling approximation, Eqs. (3.4) and (3.5).

face. Setting $\omega=2 \pi f$ and imposing the resonance condition $\Delta \Phi=2 n \pi$, we obtain the following dispersion relation for mode RPn:

$$
f=\frac{n-\left(\delta \Phi_{\text {interfaces }} / 2 \pi\right)}{2 \int_{0}^{D_{P}} \sqrt{c_{P}^{-2}-c_{H}^{-2}} d z} .
$$

Similarly, for mode $\mathrm{RS} n$ the dispersion relation is

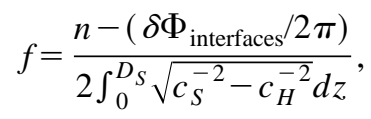

where $D_{S}$ is the depth at which $c_{S}$ first reaches $c_{H}$.

Figure 5 shows these decoupling-approximation dispersion relations for our 4-layer model of $c_{P}(z)$ and $c_{S}(z)$ (Table III). For the RS-waves, the total interface phase shift has been set to $\delta \Phi_{\text {interfaces }}=\pi$, which would be the value for a single layer with a huge rise of $c_{S}$ at its base. For the sole $\mathrm{RP}$ mode shown, RP1, it has been set to $\delta \Phi_{\text {interfaces }}=\pi / 2$, which is a fit to the dispersion relation with P-SV coupling (Fig. 6, to be discussed below).

Notice that for fixed horizontal speed $c_{H}$, the lowest RP mode, RP1, occurs at a much higher frequency $f$ than the lowest RS mode, RS1. This is because of the disparity in propagation speeds, $c_{P}=$ several $\times c_{S}$. Notice also the long, flat plateaus in $c_{H}(f)$ near $c_{H}=c_{S 2}=500 \mathrm{~m} / \mathrm{s}$ and especially $c_{S 3}=700 \mathrm{~m} / \mathrm{s}$ for the $\mathrm{RS} n$ modes, and near $c_{H}=c_{P 2}$ $=900 \mathrm{~m} / \mathrm{s}$ and $c_{H}=c_{P 3}=2400 \mathrm{~m} / \mathrm{s}$ for RP1. Mathematically these are caused by the vanishing square roots in the denomi-
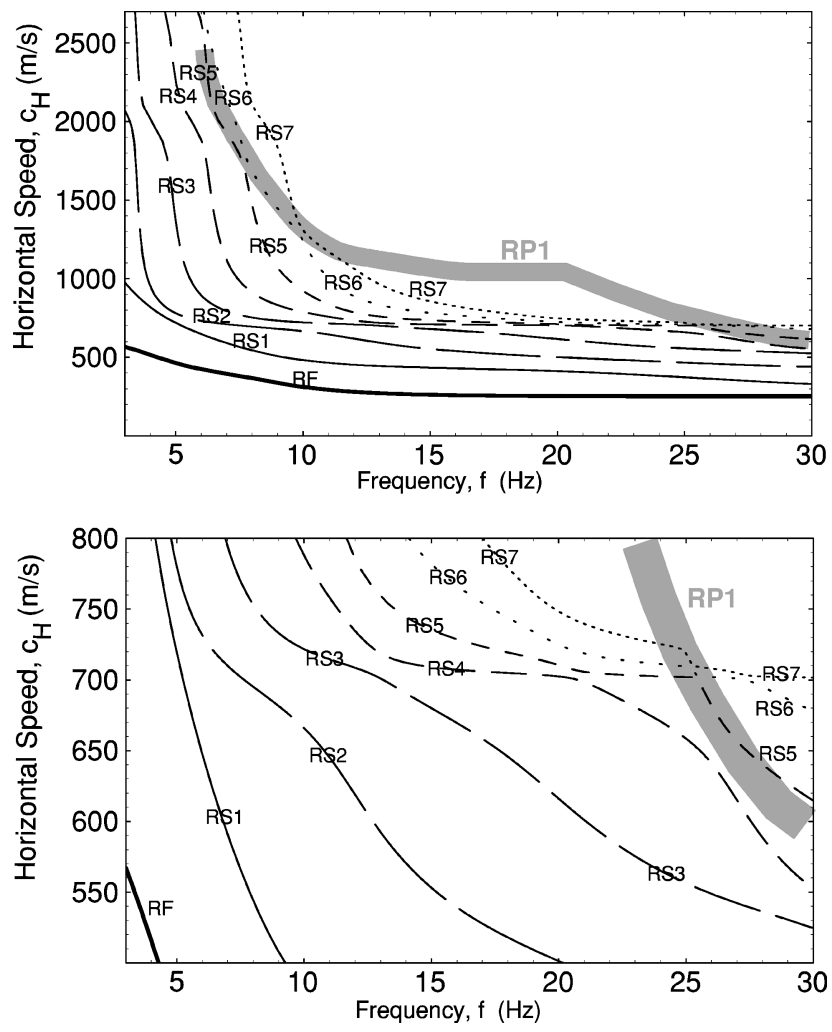

FIG. 6. Dispersion relations for the 4-layer Hanford model, including coupling between P- and SV-waves produced at boundaries between layers and at the Earth's surface.

nators of the dispersion relations (3.4) and (3.5). Physically they arise because the mode's waves "like" to propagate horizontally in their deepest layer. At high frequencies (e.g., $f \gtrsim 10 \mathrm{~Hz}$ for $c_{H} \simeq c_{S 3}=700 \mathrm{~m} / \mathrm{s}$ ), several modes propagate together nearly horizontally in that deepest layer.

\section{Effects of P-SV coupling on dispersion relations}

Figure 6 shows the dispersion relations $c_{H}(f)$ for the lowest 8 modes of our 4-layer model at Hanford, with P-SV coupling included. These dispersion relations were computed using the multilayer equations of Appendix $\mathrm{C}$. We shall now discuss these various dispersion relations, beginning with that for the fundamental mode, which is labeled RF in the figure.

Mode RF was studied in Sec. II A for an idealized homogeneous half space. It is vertically evanescent in both its Pand SV-components (except at low frequencies in the top layer); for this reason, it did not show up in our idealized decoupling-approximation dispersion relation (Fig. 5). At frequencies $f \gtrsim 10 \mathrm{~Hz}$, its vertical $e$-folding lengths $\mathcal{Z}_{P}$ and $\mathcal{Z}_{S}$ [Eqs. (2.5)] are both short enough that it hardly feels the interface between layers 1 and 2, and the homogeneous-halfspace description is rather good. Below $10 \mathrm{~Hz}$, interaction with the interface and with layer 2 pushes $c_{H}$ up.

By contrast with the P-SV-decoupled Fig. 5, every Rayleigh overtone mode RPn or RSn in Fig. 6 now contains a mixture of SV-and P-waves. This mixture varies with depth in the strata and is generated by the same kind of interface 
reflection and refraction as we met in Sec. II B for SV-up and P-up waves. In most regions of the $\left(c_{H}, f\right)$ plane, the mode mixtures are dominated either by SV- or P-waves-the ratio of energy in one wave type to that in the other is $>2$.

In the vicinity of the wide gray band marked RP1, the modes are predominately of RP type; away from that vicinity they are predominately RS. The location of the RP1 band has been inferred from the computed S- and P-wave amplitudes. Notice how well it agrees with the decoupling approximation's RP1 dispersion relation (Fig. 5). Away from the RP1 band, the dispersion relation for each RSn mode is reasonably close to its decoupling-approximation form (compare Figs. 6 and 5). As each mode nears and crosses the RP1 band, its dispersion relation is distorted to approximately coincide, for awhile, with the RP1 shape. Correspondingly, all its other properties become, for awhile, those of an RP mode.

\section{Anisotropy ratios and reduced transfer functions}

Figure 7 shows the anisotropy ratio $\mathcal{A}$ and reduced transfer function $\beta^{\prime}$ for the lowest eight modes of our 4-layer model of Hanford. These were computed using the multilayer equations of Appendix $\mathrm{C}$, with dissipation neglected. On the figure, the mode names "RSn" have been shortened to " $n$ ", and "RF" to "F." The bottom set of graphs is the value $\beta_{L}^{\prime}$ that the net reduced transfer function would have if the mode of interest were mixed with enough Love waves to reduce the net anisotropy ratio to the value $\mathcal{A}_{\text {net }} \simeq 1.0$ typical of measured seismic spectra at Hanford during quiet times [21].

Fundamental Mode RF. Above $10 \mathrm{~Hz}$, mode RF has $\mathcal{A} \simeq 2.2, \quad \beta^{\prime} \simeq 0.84$, and $\beta_{L}^{\prime} \simeq 0.58$, in accord with our homogeneous-half-space model (Sec. II). Below $10 \mathrm{~Hz}$, coupling of the RF mode to layer 2 produces a growth of the subsurface source to partially cancel the surface source, and a resulting fall of $\beta^{\prime}$ to 0.4 and $\beta_{L}^{\prime}$ to 0.35 .

RS Overtones. In RS regions (away from the RP1 band) the overtone modes $\mathrm{RS} n$ generally have $\mathcal{A} \leqq 1$ so $\beta_{L}^{\prime} \simeq \beta^{\prime}$-little or no admixed Love waves are needed to bring the anisotropy down to 1.0 . The value of $\beta^{\prime}$ ranges from $\sim 0.4$ to 1.4 in the RS regions; but when the RP1 mode is nearby in the $c_{H^{-}}-f$ plane, its admixture drives $\beta^{\prime}$ down to $\lesssim 0.2$.

Mode RS1 shows characteristic "SV-up" behavior near $25 \mathrm{~Hz}$ (compare Fig. 7 with Figs. 3 and 4). Its $\mathcal{A}$ has a very large resonance and its subsurface source (not shown in the figures) has a sharp dip to nearly zero, resulting from $45^{\circ}$ upward propagation of its SV-component in the top layer and no production of $\mathrm{P}$-waves upon reflection. At frequencies above our range of interest, this same SV-Up behavior will occur in successively higher RSn modes.

RP1 Mode. The region of RP1 behavior is shown as thick gray bands in Fig. 7 (cf. the bands in Fig. 6). The RP1 reduced transfer function is small, $\leq 0.15$, due to the same near-cancellation of its surface and subsurface sources as we met for P-Up waves in Sec. II B and Fig. 4. As each RS mode crosses the core of the RP1 region, its $\beta^{\prime}$ shows a dip and its anisotropy shows a peak, revealing the temporary transition to RP behavior.
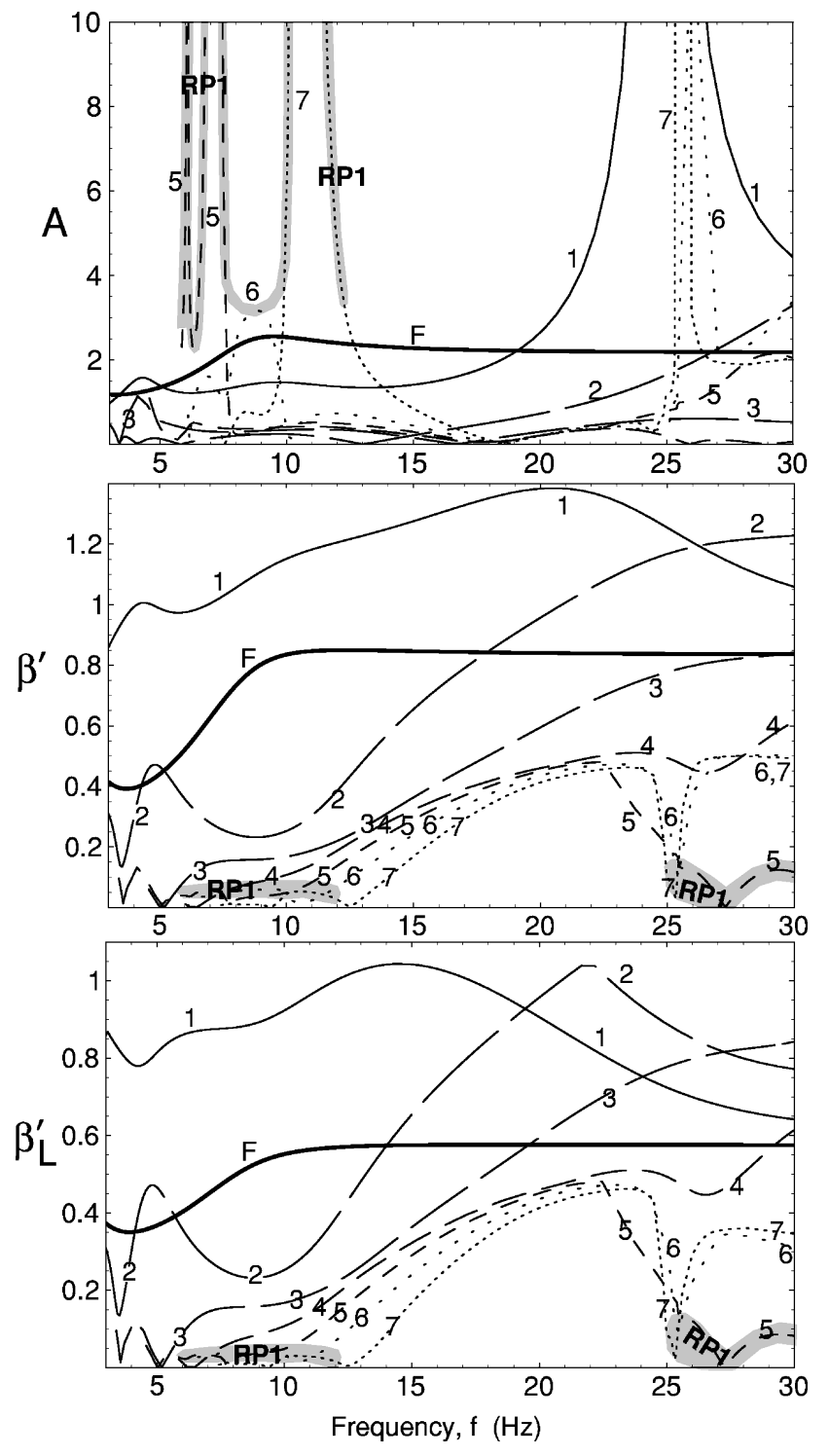

FIG. 7. Properties of the lowest 8 modes of the 4-layer Hanford model, including coupling between $\mathrm{P}$ - and $\mathrm{SV}$-waves produced at boundaries between layers and at the Earth's surface.

Higher-order RP Modes. The higher-order RP modes $(n=2,3, \ldots)$ in our frequency band will lie in the vicinity of $\mathrm{RS} n$ overtones with $n>8$. We expect these RP $n$ modes to show similarly small reduced transfer functions to those for RP1, but we have not attempted to compute them, with one important exception: high-order RP modes that travel nearly horizontally in Hanford's $\sim 4 \mathrm{~km}$ thick basalt layer. We consider these modes in the next subsection.

\section{RP modes that travel horizontally in the basalt}

As discussed in the Introduction (Sec. I D 2), the ground motions at the Hanford corner and end stations sometimes show time delays in correlated motion, corresponding to wave propagation speeds of $\sim 5000-6000 \mathrm{~m} / \mathrm{s} \quad[21,29]$. These motions must be due to wave modes that travel nearly horizontally in the $\sim 4 \mathrm{~km}$ thick basalt layer at the base of the alluvium, or in the bedrock beneath the basalt. We have 


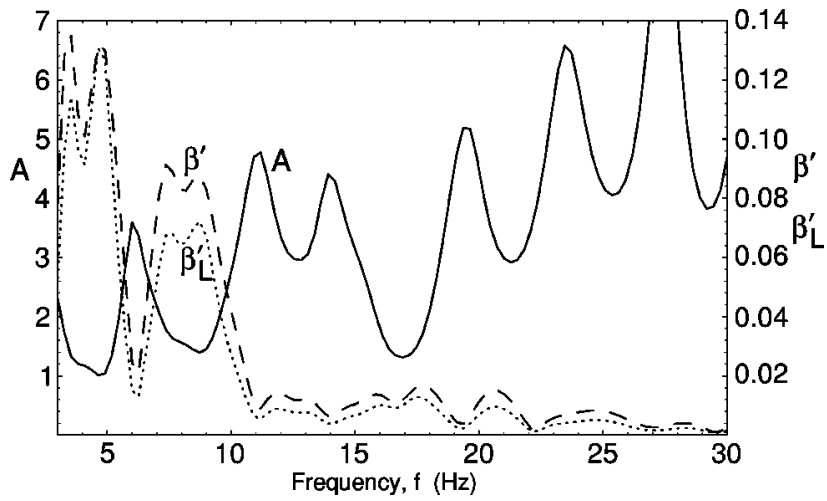

FIG. 8. Properties of RP modes that propagate nearly horizontally in layer $4\left(\sim 4 \mathrm{~km}\right.$ thick basalt layer) at Hanford $\left(c_{H}\right.$ slightly larger than $c_{P 4}=4900 \mathrm{~m} / \mathrm{s}$ ), including the effects of dissipation in the alluvium above the basalt.

computed the properties of such wave modes for the case of horizontal propagation in the basalt layer-layer 4 of our 4-layer Hanford model.

Because of the many closely spaced modes in the relevant $\left(c_{H}, f\right)$ region $\left(c_{H}\right.$ a little larger than $c_{P 4}=4900 \mathrm{~m} / \mathrm{s}$, $3 \mathrm{~Hz} \leqslant f \leqslant 30 \mathrm{~Hz}$ ), it is not reasonable, or even of interest, to compute their dispersion relations explicitly. Instead, we have assumed an idealized dispersion relation $c_{H}$ $=4910 \mathrm{~m} / \mathrm{s}$ independent of frequency.

The basalt layer is so thick that nearly horizontally propagating waves will be substantially damped in traveling from its lower face to its upper face and back; and, the S-waves will be much more strongly damped than the P-waves. For this reason, we idealize these waves as purely P-up as they impinge from the basalt layer 4 onto the layer 3-4 interface. These P-up waves at interface 3-4 are treated as a source for other wave components in all 4 layers.

For these waves, dissipation [Eqs. (1.5) and associated discussion] may be more important than for the RF, RS and RP1 modes, which were treated above as dissipationless. We therefore include it in our analysis. We do so in the 4-layer equations of Appendix $\mathrm{C}$ by giving the sound speeds appropriate imaginary parts,

$$
\begin{aligned}
& \frac{\Im\left(c_{P n}\right)}{\mathfrak{R}\left(c_{P n}\right)}=-\frac{1}{2 Q_{P}}=-0.015, \\
& \frac{\mathfrak{I}\left(c_{S n}\right)}{\mathfrak{R}\left(c_{S n}\right)}=-\frac{1}{2 Q_{S}}=-0.03,
\end{aligned}
$$

while keeping their real parts equal to the values shown in Table III. We have solved the resulting multilayer equations numerically, obtaining the anisotropy ratios and reduced transfer functions shown in Fig. 8.

The peaks in $\mathcal{A}$ at $f \simeq 3,11,19$, and $27 \mathrm{~Hz}$ [frequency separation $\left.\Delta f=c_{P 3} /\left(2 D_{3} \sqrt{1-c_{P 3}^{2} / c_{H}^{2}}\right)=8 \mathrm{~Hz}\right]$ are associated with resonant $\mathrm{P}$-wave excitations of layer 3 and their influence on layer 2 and thence on layer 1 ; $c f$. the decoupling-approximation dispersion relation (3.4). The slightly smaller peaks at $f \simeq 6,14$, and $23 \mathrm{~Hz}$ are due to resonant $\mathrm{S}$-wave excitations of layer 2 . The oscillations in both $\mathcal{A}$ and (less obviously) $\beta^{\prime}$ with frequency $\Delta f$ $=c_{P 2} /\left(2 D_{2} \sqrt{1-c_{P 2}^{2} / c_{H}^{2}}\right)=16 \mathrm{~Hz}$ are associated with resonant $\mathrm{P}$-wave excitations of layer 2.

Despite the complexity of these deeply seated RP modes, with various types of resonant excitations of various layers, and despite the fact that the seismic gravity gradients arise from depths $\mathcal{Z}_{\mathrm{sgg}}=1 / k \sim 25$ to $250 \mathrm{~m}$ so great that the top three layers all contribute, these modes exhibit the same range of values of $\beta^{\prime}$ as mode RP1: 0 to 0.15 . Here, as for RP1 and for P-up waves in a homogeneous half space, $\beta^{\prime}$ is small because of near cancellation of the gravity gradients from the P-wave surface and subsurface sources.

\section{Summary of Hanford model results}

The most important of the above results are those for the reduced transfer functions of the various modes at Hanford. They are summarized in Table I and their implications are discussed in the Introduction, Sec. I D 2.

\section{LIVINGSTON}

\section{A. Livingston geophysical structure}

At the LIGO site near Livingston, Louisiana, the geological strata consist of alluvial deposits laid down by water flowing into the Gulf of Mexico. As the ocean level has risen and fallen, alluvial terraces of varying thickness have been formed. This alluvium (layers of clay, silt, sand, and gravel in various orders) is of the Holocene, Pleistocene, and Pliocene eras going down to a depth of about $700 \mathrm{~m}$, and compacted alluvium of the upper Miocene and earlier eras below that. These sedimentary deposits extend down to a depth of about $3 \mathrm{~km}$ [19] before reaching bedrock.

For our analysis the principal issue is the vertical velocity profiles $c_{P}(z)$ and $c_{S}(z)$. The primary difference between Livingston and Hanford is the depth of the water table: it is only about $2 \mathrm{~m}$ down at Livingston, versus about $40 \mathrm{~m}$ at Hanford. This difference should cause $c_{P}$ to soar to about $1600 \mathrm{~m} / \mathrm{s}$ at depths of a few meters at Livingston; it only does so roughly $40 \mathrm{~m}$ down at Hanford.

The only measurements of the Livingston velocity profiles that we have been able to find are those performed in a site survey for LIGO [34]. Those measurements only include $c_{S}$, not $c_{P}$, and only go down to a depth of $15 \mathrm{~m}$. Accordingly, we have had to estimate the velocity profiles from these sparse data and from the lore accumulated by the geophysics and seismic engineering communities.

That lore suggests that $c_{S}$ should increase as about the 1/4 power of depth [18]. (This increase is due to the fact that the shear restoring force must be carried by the small-area interfaces between the grains of gravel, sand, silt, or clay; the weight of overlying material compacts the grains, increasing the areas of their interfaces.) We have fit the measured $c_{S}(z)$ in the top $15 \mathrm{~m}(\{7 \mathrm{ft}, 700 \mathrm{ft} / \mathrm{s}\},\{21 \mathrm{ft}, 810 \mathrm{ft} / \mathrm{s}\},\{50 \mathrm{ft}, 960$ $\mathrm{ft} / \mathrm{s}\}$ ) (Ref. [34], Appendix B, plate 7) to a $1 / 4$ power law, adjusting the fit somewhat to give speeds at greater depths in rough accord with measurements at a similar sedimentary site in Tennessee [35]. Our resulting fit is

$$
c_{S}=185 \mathrm{~m} / \mathrm{s}(1+z / 2.9 \mathrm{~m})^{1 / 4} \text {. }
$$


TABLE IV. Four-layer model for the velocity profiles at the Livingston LIGO site. Notation and units are as in Table III.

\begin{tabular}{llrrrc}
\hline \hline$n$ & \multicolumn{1}{c}{ Depths } & \multicolumn{1}{c}{$D_{n}$} & $c_{P n}$ & $c_{S n}$ & $\nu_{n}$ \\
\hline 1 & $0-5$ & 5 & 440 & 220 & 0.33 \\
2 & $5-105$ & 100 & 1660 & 400 & 0.47 \\
3 & $105-905$ & 800 & 1700 & 700 & 0.40 \\
4 & $905-3005$ & 2100 & 1900 & 1000 & 0.31 \\
\hline \hline
\end{tabular}

A combination of theory and phenomenology [Eqs. (6.24), (6.26) of [18] and associated discussion] tells us that in these water-saturated alluvia, the material's Poisson ratio should be about

$$
\nu=\frac{1}{2}\left[1-0.39\left(\frac{c_{S}}{1000 \mathrm{~m}}\right)^{2}\right]
$$

(The Poisson ratio goes down gradually with increasing compaction and increasing $c_{S}$ because water is playing a decreasing role compared to the grains.) The standard relation

$$
c_{P}=c_{S} \sqrt{\frac{2-2 \nu}{1-2 \nu}}
$$

combined with Eqs. (4.1a) and (4.1b), then gives us the vertical profile for $c_{P}$.

These profiles are valid only in the water-saturated region. Although the water table is at $\sim 2 \mathrm{~m}$, measurements elsewhere [35] suggest that one may have to go downward an additional several meters before the effects of the water on $c_{P}$ will be fully felt. Accordingly, we expect $c_{P} \sim 2 c_{S}$ in the top $\sim 5 \mathrm{~m}$ at Livingston, followed by a sharp rise to the values dictated by Eqs. (4.1a)-(4.1c), though in our final conclusions (Sec. IV C 2), we shall allow for the possibility that the sharp rise occurs at anywhere from 2 to $5 \mathrm{~m}$ depth.

\section{B. Livingston 4-layer model}

We have fit a four-layer model to these estimated Livingston velocity profiles. Our fit is shown in Table IV. This model is the primary foundation for our exploration of seismic gravity gradients at Livingston. As discussed above, it principally differs from the 4-layer Hanford model by the rapid increase of $c_{P}$ at $5 \mathrm{~m}$ depth at Livingston, due to the higher water table. All other differences have a much more minor influence on the seismic gravity-gradient noise.

\section{Livingston model results}

\section{Mode overview}

Because the top, unsaturated layer is so thin, RP modes cannot resonate in it in our frequency band; and because water makes $c_{P}$ so large just below the top layer, the RP modes in our band can only propagate at a correspondingly high speed, $c_{H}>1660 \mathrm{~m} / \mathrm{s}$. The lowest $10 \mathrm{RS}$ modes, by contrast, are confined to speeds $c_{H} \lesssim 1000 \mathrm{~m} / \mathrm{s}$. As a result-in contrast to Hanford-there is no mixing between

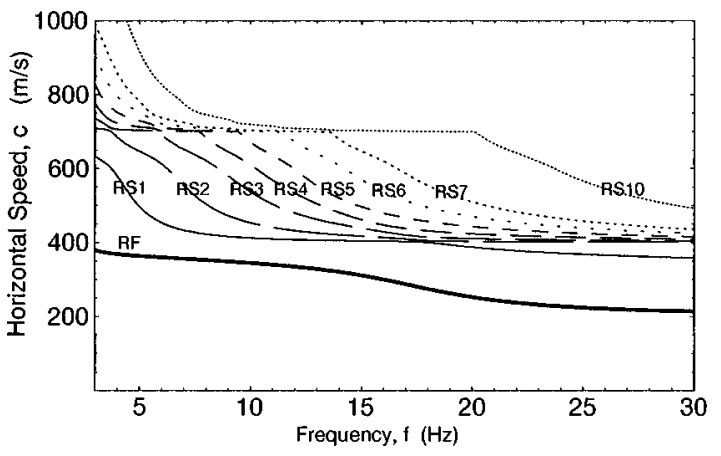

FIG. 9. Dispersion relations for 4-layer Livingston model, including coupling between $\mathrm{P}$ - and SV-waves produced at boundaries between layers, for the fundamental mode and the lowest 10 RS modes.

these lowest RS modes and the RP modes. The RS modes have purely RS character, with no significant RP admixture.

In the next section we shall study the lowest 10 RS modes along with the fundamental mode. In the following section, we shall examine the lowest RP mode.

\section{2. $R F$ and $R S$ modes}

We have computed the dispersion relations, anisotropy ratios, and reduced transfer functions for modes RF and RS1-10 in our 4-layer Livingston model, using the multilayer equations of Appendix $\mathrm{C}$. The dispersion relations are shown in Fig. 9. Because of the separation in the $\left(c_{H}, f\right)$ plane of these modes from the RP mode, we expect the P-SV decoupling approximation to work quite well here. Indeed, the RS modes have the form one would expect from the decoupling approximation [Eqs. (3.5)]. The anisotropy ratios and reduced transfer functions are shown in Fig. 10.

RF Mode. Because the top layer is 2.5 times thinner in our Livingston model than at Hanford, the frequency at which the RF mode becomes like that of a homogeneous half space is 2.5 times higher: $\sim 25 \mathrm{~Hz}$ compared to $\sim 10 \mathrm{~Hz}$. Only above $\sim 25 \mathrm{~Hz}$ do the mode's properties asymptote toward their homogeneous-half-space values of $c_{H}=205 \mathrm{~m} / \mathrm{s}$, $\mathcal{A}=2.2, \beta^{\prime}=0.86$ and $\beta_{L}^{\prime}=0.40$. At lower frequencies, interaction with layer 2 pushes $\beta^{\prime}$ into the range $0.65-0.9$, and $\beta_{L}^{\prime}$ into the range $0.35-0.45$.

It is possible that the effects of water saturation will cause $c_{P}$ to shoot up at depths shallower than the $5 \mathrm{~m}$ assumed in our model; a transition anywhere in the range $2 \mathrm{~m} \lesssim z \lesssim 5 \mathrm{~m}$ must be considered reasonable. If the transition in fact occurs at depths shallower than $5 \mathrm{~m}$, the peaks of $\beta^{\prime}$ and $\beta_{L}^{\prime}$ will be pushed to correspondingly higher frequencies. Thus, we must be prepared for the RF mode to have $\beta^{\prime}$ anywhere in the range $0.65-0.9$, and $\beta_{L}^{\prime}$ in the range $0.35-0.45$ at just about any frequency in our band of interest.

RS Modes. In our frequency band, the RS modes have negligible excitation in layers 3 and 4, and their P-waves are evanescent in layers 2, 3 and 4. As a result, these modes can be well approximated by SV-up waves in layer 2, impinging on the layer 1-2 interface. We have verified this by computing their anisotropies and reduced transfer functions in this 

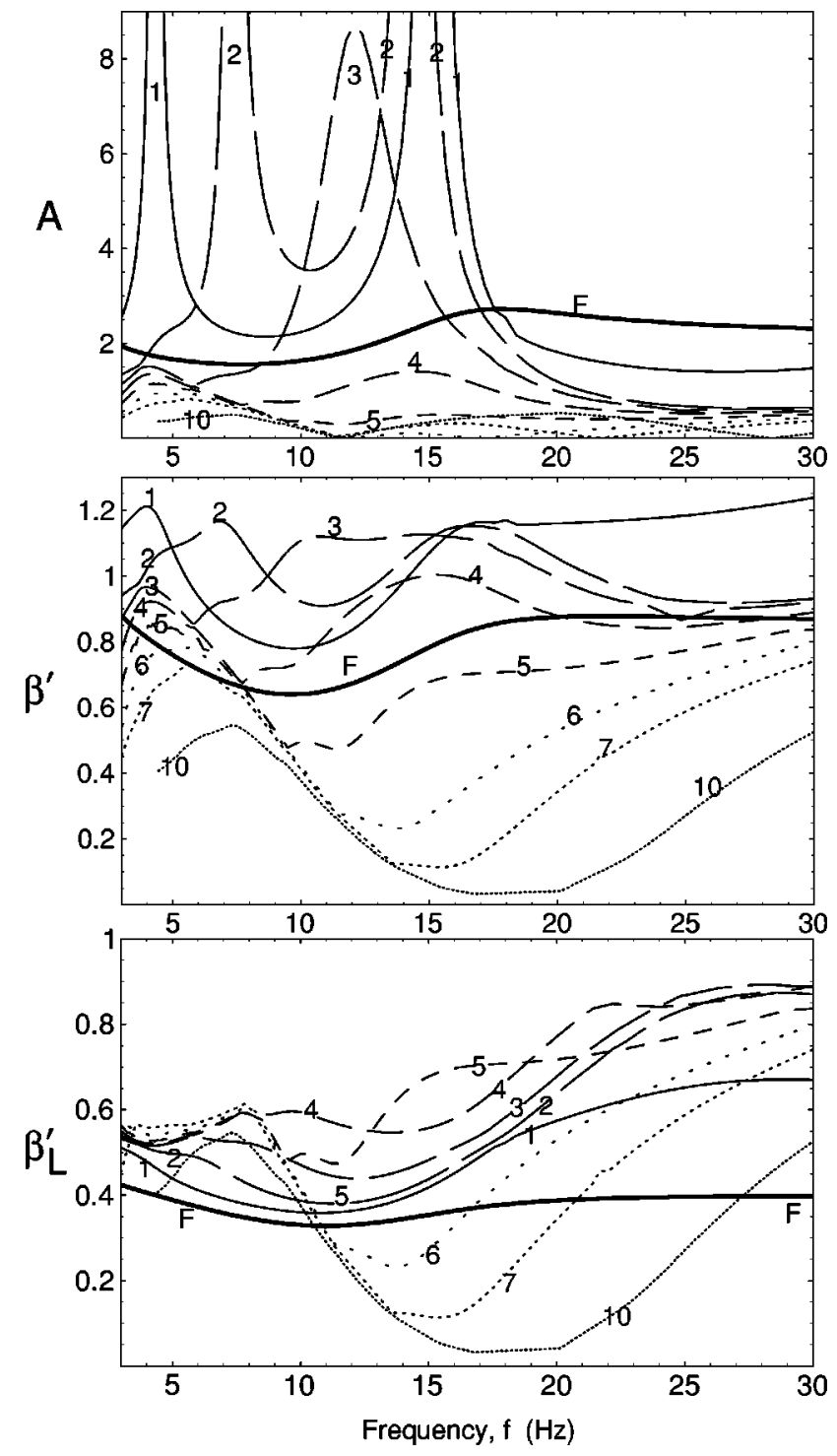

FIG. 10. Properties of the lowest $10 \mathrm{RS}$ modes and the RF mode of the 4-layer Livingston model. (Modes RS8 and RS9 are not shown; their curves are sandwiched between 7 and 10.)

2-layer SV-up approximation by the method outlined at the beginning of Appendix E. The results for $\mathcal{A}$ and $\beta^{\prime}$, which relied on the 4-layer dispersion relations of Fig. 9, agree to within a few per cent with those of our 4-layer model (Fig. 10) except at frequencies below $5 \mathrm{~Hz}$ where the differences become somewhat larger.

Throughout our frequency band these RS modes have vertical seismic-gravity-gradient $e$-folding lengths $\mathcal{Z}_{\text {sgg }}=1 / k$ $\gtrsim D_{1}=5 \mathrm{~m}$. Thus, the upper parts of layer 2 contribute significantly to the reduced transfer function $\beta^{\prime}$, along with all of layer 1 .

For modes RS1-RS5, the gravity gradients are largely due to the S-waves' vertical surface motions, and correspondingly the reduced transfer functions have the familiar range $\beta^{\prime} \simeq 0.6-1.2$ that we encountered for RS modes at Hanford (Sec. III B 3) and for SV-Up modes in a homogeneous half space (Fig. 4).

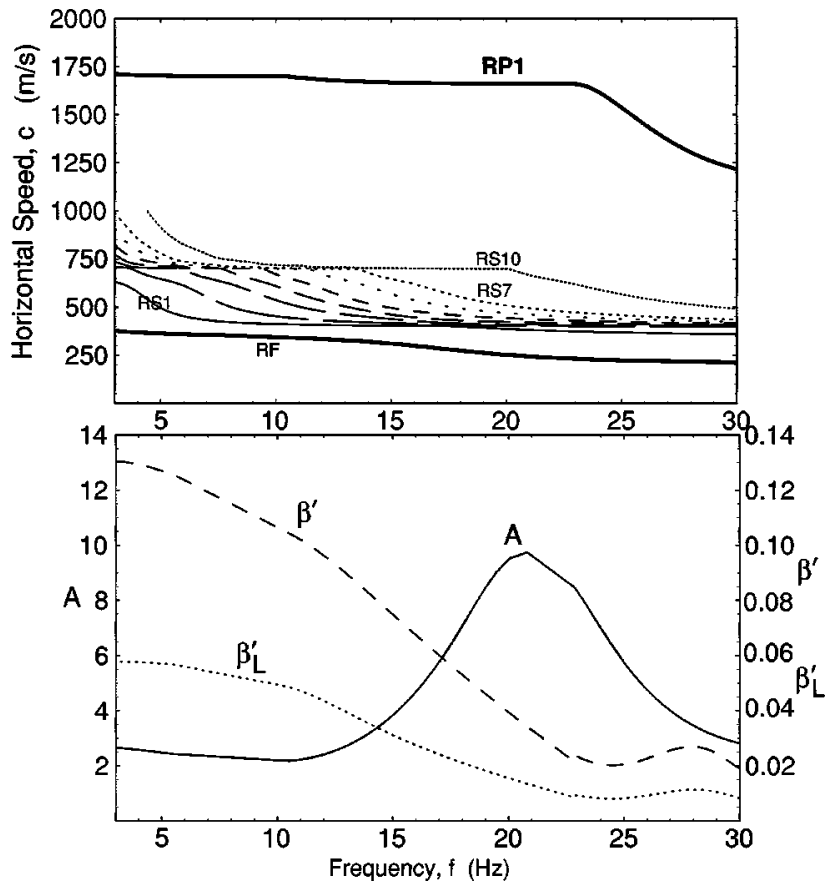

FIG. 11. Upper panel: dispersion relation for mode RP1 in the 4-layer Livingston model. Also shown for comparison are the dispersion relations for the fundamental Rayleigh mode RF and the lowest few RS modes (cf. Fig. 9). Lower panel: properties of mode RP1.

By contrast, modes RS6-RS10 show a phenomenon not exhibited at Hanford: a broad dip in $\beta^{\prime}$ to a value $\ll 1$. This dip is caused by a significant excitation of P-waves in Livingston's 5-meter thick top layer: the vertical surface motions in the dip are largely due to the P-waves, and mass conservation guarantees that the gravity gradients they produce will be nearly cancelled by those from the subsurface, P-wave compressional source. These surface-layer excitations are not associated with any RP mode; as we shall see in the next subsection, the lowest RP mode at these frequencies has $c_{H}$ about twice as high as for these modes. It seems that the close proximity of the two very sharp geophysical discontinuities (the earth's surface and the sharp rise of $c_{P}$ caused by water) forces the modes' $\mathrm{S}$-waves to generate a sizable component of P-waves even moderately far from Pwave resonance. No such phenomenon was observed in our Hanford 4-layer model.

\section{Mode RPI}

Figure 11 shows the dispersion relation for the lowest RP mode, RP1, at Livingston, along with the RF and lowest 10 RS modes. As noted earlier, RP1 does not overlap the other modes [by contrast with Hanford (Figs. 5 and 6)].

At frequencies $f<22.8 \mathrm{~Hz}$, the RP1 mode has horizontal speed $c_{H}>c_{P 2}=1660 \mathrm{~m} / \mathrm{s}$ and thus its P-waves can propagate in layers 1 and 2 (and also in layer 3 below $11.3 \mathrm{~Hz}$ ). In this region we have evaluated $c_{H}(f)$ using the P-SV decoupling approximation [Eq. (3.4)].

At frequencies $f>22.8 \mathrm{~Hz}$, the horizontal speed is $c_{H}$ $<c_{P 2}$, so the mode's P-waves are evanescent in layers 2,3 , 
and 4. In this regime we have adopted an approximation that is much more accurate than the decoupling one. We have idealized the material as two-layered: a $5 \mathrm{~m}$ thick upper layer with the properties of layer 1 of Table IV, and below that a homogeneous half space with the properties of layer 2. For this layer-plus-half-space model we have used an analytic dispersion relation due to Lee [36] (Appendix D). Because the mode's SV-waves can leak out of layer 1 into layer 2 (and then propagate away to "infinity" - or, more realistically, dissipate), Lee's dispersion relation predicts a complex frequency $f$ if $c_{H}$ is chosen real, and a complex $c_{H}$ if $f$ is chosen real. The predicted losses are small (quality factors $Q$ decreasing from $\simeq 50$ at $f \simeq 24 \mathrm{~Hz}$ to $\simeq 15$ at $30 \mathrm{~Hz}$ ). The real part of the dispersion relation is shown in the upper panel of Fig. 11.

The lower panel of Fig. 11 shows the anisotropies and reduced transfer functions for this RP1 mode. At $f$ $<22.8 \mathrm{~Hz}$, where the $\mathrm{P}$-waves are propagating nearly horizontally in layer 2, these properties were computed using the P-up approximation in the above two-layer (layer-plus-halfspace) model; $c f$. the introduction to Appendix E. More specifically, the dispersion relation (with both $c_{H}$ and $f$ real) was taken from the P-SV decoupling approximation, the $\mathrm{P}$ waves with this $c_{H}$ and $f$ were regarded as impinging from layer 2 onto the top of layer 1 at a glancing angle, and the reflected $\mathrm{P}$ - and $\mathrm{SV}$-waves were regarded as propagating off to "infinity" (or, more realistically, dissipating before any return to the interface). This is the approximation that was so successful for the RS modes when combined with the correct 4-layer dispersion relation, but we don't have a good handle on its accuracy here, with the less reliable P-SV-decoupling dispersion relation. We are much more confident of our approximation for $f>22.8 \mathrm{~Hz}$. There we used the exact twolayer equations (Appendix C), together with Lee's exact, complex dispersion relation $c_{H}(f)$.

These computations produced an anisotropy that peaks at $f=22.8 \mathrm{~Hz}$ where $c_{H}=c_{P 2}$, with a peak value of $\mathcal{A} \sim 8$ (Fig. 11). This is smaller than the peak anisotropies for mode RP1 at Hanford (Fig. 7), but comparable to those for the higherorder RP modes that propagate nearly horizontally in the Hanford basalt (Fig. 8).

The reduced transfer function $\beta^{\prime}$ lies in the same range, 0 to 0.15 , as for all the Hanford RP modes that we studied (Figs. 7 and 8). This adds to our conviction that this low range of $\beta^{\prime}$ is a general characteristic of RP modes.

\section{Summary of Livingston model results}

The most important of the above results are those for the reduced transfer functions $\beta^{\prime}$ of the various modes at Livingston. They are summarized in Table I and their implications are discussed in the Introduction, Sec. I D 2.

\section{CONCLUDING REMARKS}

\section{A. Summary}

In this paper, we have used the theory of seismic surface waves to calculate the seismic gravity-gradient noise spectra that are to be expected at the Hanford, Washington and Liv- ingston, Louisiana LIGO sites. Our final noise strengths, as shown in Fig. 2, are close to Saulson's previous rough estimate. At noisy times and near $10 \mathrm{~Hz}$, the seismic gravitygradient noise is likely to be more serious than vibrational and thermal seismic noise in advanced interferometers. Unless means are found to combat gravity-gradient noise (see below for possible methods), the hard-won gains in sensitivity due to $R \& D$ on vibration isolation and thermal noise may be compromised by seismic gravity gradients, at least at noisy times.

\section{B. Effects of topography and of LIGO construction}

In our analysis we have idealized the Earth's surface near the LIGO test masses as perfectly planar and as undisturbed by LIGO construction. Irregularities in topography will significantly disturb the waves' propagation and their vertical structure only if the surface height varies by amounts as large as $\sim 2 \mathrm{~m} /(f / 10 \mathrm{~Hz})=(\sim 1 / 2$ the shortest vertical $e$ - folding length $\mathcal{Z}_{P}$ for $\mathrm{RF}$ waves), on horizontal lengthscales as short as $\sim 8 \mathrm{~m} /(f / 10 \mathrm{~Hz})=(\sim 2$ times the horizontal reduced wavelength $1 / k$ of those RF waves), within distances of the test masses $\sim 25 \mathrm{~m} /(f / 10 \mathrm{~Hz})=$ (the horizontal wavelength of those RF waves), for frequencies $\sim 3-30 \mathrm{~Hz}$. (Of all the modes we have studied, the RF modes hug the surface most tightly and thus will be most influenced by the topography.)

Variations on these scales were rare at the two LIGO sites before construction. However, the grading that made the arms flat produced topographic variations in the vicinity of some of the test masses that are marginally large enough to disturb the propagation. Examples are the long pits dug alongside the arms at Livingston to get material for building up the arms' heights, and excavation at Hanford to lower the arms below the level of the surrounding land near the southwest arm's midstation and the northwest arm's endstation.

We speculate that these topographic modifications will alter the seismic gravity gradient noise by a few tens of percent, but probably not by as much as a factor 2 . Future studies should examine this issue.

The $1 \mathrm{~m}$ deep concrete foundations of the buildings that house the test masses will likely also influence the noise by a few tens of percent, particularly at $\sim 20-30 \mathrm{~Hz}$ where the RF waves' vertical penetration is short. The foundation extends approximately 10 meters by 25 meters at the interferometer's end stations (and also, in the case of Hanford, at the mid station). The foundation is approximately " $\mathrm{X}$ " shaped for the corner stations, with each arm of the " $\mathrm{X}$ ' extending roughly 100 meters by 20 meters [37]. The sound speeds in the concrete will be a factor of several higher than the surrounding ground, so the foundations will form very sharp "geophysical" interfaces in the ground, causing diffraction of impinging waves and altering their vertical structure. Because the foundations are so shallow, we doubt that their net effect on the seismic gravity gradient noise can be as large as a factor 2, but future studies should examine it.

\section{Measurements that could firm up our understanding of seismic gravity gradients}

Our analysis is plagued by a large number of uncertainties regarding the true make-up of the ambient seismic back- 
ground at the LIGO sites. We made extensive use of measurements of ground motion which functioned as constraints on what modes could be present. These measurements were helpful, but certain other measurements would be considerably more helpful. We suggest that, to the extent that resources permit, these measurements be included in future seismic surveys for gravitational-wave interferometer sites, including future surveys at the LIGO sites.

First, we recommend careful measurements of the sound speeds and dynamical Poisson ratios of the ground as a function of depth, especially in the top few tens of meters and if possible down to the bedrock. At Hanford, we had reasonably complete data [33], thanks to earlier plans to build a nuclear power plant in the vicinity. As discussed in this paper, we encountered serious discrepancies between those old data and data from the LIGO geotechnical survey. At Livingston, we had no P-wave speed or Poisson ratio profiles, and the S-wave speed profiles available only went down to a depth of 15 meters. As a result, we had to use a mixture of theory, profiles from other sites, and phenomenological fitting to obtain a plausible velocity profile. Velocity profiles are of crucial importance in determining how the various modes behave in the ground.

Second, we recommend measurements that more nearly directly determine the modes that characterize the seismic motion. In this paper, as discussed above, we were able to put together very rough estimates of the modes that actually characterize the seismic background by using surface motion data as constraints, particularly anisotropy ratios measured at the sites, and by appealing to more detailed measurements at other sites. However, other techniques could provide much more useful and restrictive constraints, thereby more sharply differentiating among the various modes. In particular.

Surface seismic arrays $[28,38]$ allow one to measure the phase relationships of ground motion at appropriately separated points, from which one can infer the excited modes' wave numbers $k(f)$ and horizontal propagation speeds $c_{H}(f)$.

Borehole measurements [28] allow one to measure the phase correlation of motion at the surface and at some depth $z$ underground, and the variation of amplitudes with depth, thereby introducing additional constraints on the background.

Specialty seismic instruments called "dilatometers" $[39,40]$ measure directly the fractional density perturbation $\delta \rho / \rho$ that is the subsurface source of seismic gravity gradients. Measurements down boreholes with such devices could place further constraints on the mode mixtures present, and could show how $\delta \rho / \rho$ varies with depth, at fixed frequency. When correlated with vertical surface seismic measurements, they could give information about the cancellation of gravity gradients from the surface and subsurface sources.

\section{Mitigation of seismic gravity gradient noise}

Seismic gravity gradients are unlikely to be a major concern to LIGO detectors in the near future, since these detectors are only sensitive to frequencies $f \geq 35 \mathrm{~Hz}$. Eventually, however, LIGO experimenters may succeed in achieving ex- tremely good vibration isolation and thermal noise control at frequencies $f \leqq 10 \mathrm{~Hz}$. At this time, the detectors may well be plagued by seismic gravity-gradient noise, at least at noisy times; and there may be a strong need to try to mitigate it.

We see two possibilities for modest amounts of mitigation: (i) monitoring the noise and removing it from the LIGO data, and (ii) building moats to impede the propagation of RF-mode seismic waves into the vicinities of the test masses.

Monitoring and correction: By using dedicated 3dimensional arrays of vertical surface seismometers and borehole-mounted dilatometers in the vicinities of all test masses, one might be able to determine both the surface and subsurface components of $\delta \rho / \rho$ with sufficient spatial and temporal resolution for computing the seismic gravity gradient noise and then removing it from the data. This paper's insights into the modes at the two LIGO sites and the gravity gradients they produce may provide a foundation for future explorations of monitoring-and-correction strategies.

Moats: By constructing a narrow, evacuated moat around each test mass, one might succeed in shielding out a significant portion of the RF waves that we suspect are the dominant source of quiet-time seismic gravity gradients. Since the $\mathrm{RF}$ mode contains substantial S-waves and they are the dominant contributors to the gravity-gradient noise, such moats may have to be at least as deep as the S-waves' vertical e-folding length, $\mathcal{Z}_{S} \simeq 9-15 \mathrm{~m}(10 \mathrm{~Hz} / f)$ [Eq. (2.5) modified for an increase in the RF speed $c_{H}$ due to stratification as shown in Figs. 6 and 9]. Since $\mathcal{Z}_{S} \simeq 2.5 \mathcal{Z}_{P}$, moats of this depth would strongly shield out the RF mode's Pwaves.

The radius of the moats should be $\geq \lambda \sim 20$ $-35 \mathrm{~m}(10 \mathrm{~Hz} / f)$. It is not clear to us whether such moats at Livingston would be effective if filled with water, or whether they would have to be kept pumped out. The water would shield out the RF mode's S-waves but transmit its P-waves. If, after transmission, the waves remain mostly of P-type, then a significant reduction of $\beta^{\prime}$ could result; but it is not at all obvious how much regeneration of $\mathrm{S}$-waves would occur in the moat-surrounded cavity. Detailed modeling would be required to sort out such issues.

Although moats may be well-suited to reduce gravity gradients generated by the RF mode, they are probably not so well-suited to reduce gravity gradients generated by Rayleigh overtones. The overtones can be visualized as seismic waves that propagate by bouncing between layer interfaces and the Earth's surface; they could propagate right under the moat and into the region under the test mass. Conceivably, they could even resonantly "ring" the earth under the mass, worsening the seismic gravity-gradient noise.

If seismic gravity gradients become a problem in the future, ideas such as moats and monitoring-and-correcting will have to explored.

\section{ACKNOWLEDGMENTS}

We thank Peter Saulson for triggering this research, and Kenneth Libbrecht, Rai Weiss and Stan Whitcomb for helpful comments. We thank geophysicists Hiroo Kanamori and 
Susan Hough and seismic engineer Ronald Scott for very helpful conversations and advice, and Alan Rohay for advice and for providing his measurements of the seismic ground motion at Hanford and Livingston. We thank Albert Lazzarini for facilitating access to Rohay's data sets and to blueprints of the LIGO site facilities, and Fred Asiri for helping us to track down information about the geological structures at the sites (including the Skagit report). We also thank Saul Teukolsky for pointing out an analytic substitution which simplified our formula for $\gamma(y)$. Finally, we thank Giancarlo Cella for providing us, shortly before this paper was submitted, a copy of the VIRGO-Project manuscript on seismic gravity gradient noise [12] and for a helpful discussion. This research was supported by NSF Grant PHY-9424337. S.A.H. gratefully acknowledges the support of the National Science Foundation Graduate Program. K.S.T. thanks the Max-Planck-Institut für Gravitationsphysik for hospitality during the final weeks of writing this manuscript.

\section{APPENDIX A: GENERAL EXPRESSION FOR REDUCED TRANSFER FUNCTION}

In this Appendix we derive Eqs. (1.17)-(1.24) for the reduced transfer function and anisotropy ratio of an arbitrary Rayleigh mode. In the text the mode is labeled $J$; in this Appendix we shall omit the subscript $J$.

The mode has frequency $f$, angular frequency $\omega=2 \pi f$, horizontal wave number $k$, horizontal phase speed $c_{H}$ $=\omega / k$, and horizontal propagation direction $\hat{k}$. At the earth's surface its displacement vector is

$$
\vec{\xi}(z=0)=\left(\xi_{H} \hat{k}-\xi_{V} \vec{e}_{z}\right) e^{i(\vec{k} \cdot \vec{x}-\omega t)}
$$

[Eq. (1.21)]; and on and beneath the surface it produces a fractional density perturbation

$$
\frac{\delta \rho}{\rho}=\left[\xi_{V} \delta(z)+\mathcal{R}(z)\right] e^{i(\vec{k} \cdot \vec{x}-\omega t)}
$$

[Eq. (1.22)]; here $\vec{k}=k \hat{k}$ is the horizontal wave vector and $\delta(z)$ is the Dirac delta function.

Since the ambient seismic motions are horizontally isotropic, this mode is excited equally strongly for all horizontal directions $\hat{k}$, and also for all wave numbers in some (arbitrarily chosen) small band $\Delta k$ around $k$-i.e., in the annulus $\mathcal{C}_{\Delta k}$ of width $\Delta k$ in wave-vector space. Correspondingly (with an arbitrary choice for the strength of the excitation), the net displacement along some horizontal direction $\hat{n}$, in the frequency band $\Delta f=c_{H} \Delta k / 2 \pi$, is

$$
X(t)=\mathfrak{R}\left[\sum_{\vec{k}} \xi_{H}(\hat{k} \cdot \hat{n}) e^{i(\vec{k} \cdot \vec{x}-\omega t)}\right],
$$

and the power of this random process $X(t)$ in the frequency band $\Delta f$ is

$$
\widetilde{X}^{2}(f) \Delta f=\sum_{\vec{k}}\left|\xi_{H}\right|^{2}(\hat{k} \cdot \hat{n})^{2}=\left|\xi_{H}\right|^{2} \frac{N_{\Delta \mathrm{k}}}{2},
$$

where $N_{\Delta \mathrm{k}}=\sum_{\vec{k}} 1$ is the (normalization-dependent) total number of allowed $\vec{k}$ values in the annulus $\mathcal{C}_{\Delta k}$, and the $1 / 2$ comes from averaging $(\hat{k} \cdot \hat{n})^{2}$ over the horizontal direction $\hat{k}$. (Note: the overall normalization $N_{\Delta k}$ of our procedure for going from the random process expressed as a sum over directions to the processes's power will have no influence on our final answers for $\mathcal{A}$ and $\beta^{\prime}$, since they are square roots of ratios of powers from which $N_{\Delta k}$ drops out.) Similarly, the net displacement and power along the vertical $\vec{e}_{z}$ direction are

$$
Z(t)=\Re\left[-\sum_{\vec{k}} \xi_{V} e^{i(\vec{k} \cdot \vec{x}-\omega t)}\right]
$$

and

$$
\widetilde{Z}^{2}(f) \Delta f=\sum_{\vec{k}}\left|\xi_{V}\right|^{2}=\left|\xi_{V}\right|^{2} N_{\Delta \mathrm{k}} .
$$

The mode's anisotropy ratio, $\mathcal{A}=\widetilde{Z} / \widetilde{X}$ is therefore

$$
\mathcal{A}=\sqrt{2}\left|\xi_{V}\right| /\left|\xi_{H}\right|
$$

cf. Eq. (1.23); and the direction-averaged power $\widetilde{W}^{2} \Delta f$ $=\left(2 \widetilde{X}^{2} \Delta f+\widetilde{Z}^{2} \Delta f\right) / 3$ is

$$
\widetilde{W}^{2} \Delta f=\frac{\left|\xi_{H}\right|^{2}+\left|\xi_{V}\right|^{2}}{3} N_{\Delta k}
$$

By analogy with Eq. (A3), the isotropically excited mode produces a fractional perturbation in density on and beneath the earth's surface given by

$$
\frac{\delta \rho}{\rho}=\mathfrak{R}\left[\sum_{k}\left[\xi_{V} \delta(z)+\mathcal{R}\right] e^{i(\vec{k} \cdot \vec{x}-\omega t)}\right]
$$

cf. Eq. (A2). As an aid in computing the gravitational acceleration produced on one of the interferometer's test masses by these density perturbations, we place the origin of coordinates (temporarily) on the earth's surface, immediately beneath the test mass. Then the location of the test mass is $-\mathcal{H} \vec{e}_{z}$, where $\mathcal{H}$ is its height above the surface. We denote by $\hat{m}$ the unit vector along the laser beam that is monitoring the test mass's position. Then the gravitational acceleration along the $\hat{m}$ direction is

$$
a_{\hat{m}}(t)=-\int d^{3} x^{\prime} \frac{\left(\vec{x}^{\prime} \cdot \hat{m}\right) G \delta \rho\left(\vec{x}^{\prime}, t\right)}{\left|\vec{x}^{\prime}+\mathcal{H} \vec{e}_{z}\right|^{3}} .
$$

Invoking Eq. (A9) and introducing Cartesian coordinates $\left(x^{\prime}, y^{\prime}, z^{\prime}\right)$ inside the sum with $\vec{k}$ along the $x^{\prime}$-direction, we bring Eq. (A10) into the form 


$$
\begin{aligned}
a_{\hat{m}}= & -\sum_{\vec{k}} e^{-i \omega t} G \rho \\
& \times \iiint \frac{\left(x^{\prime} m_{x}+y^{\prime} m_{y}\right) e^{i k x^{\prime}}\left[\xi_{V} \delta\left(z^{\prime}\right)+\mathcal{R}\left(z^{\prime}\right)\right]}{\left[x^{\prime 2}+y^{\prime 2}+\left(z^{\prime}+\mathcal{H}\right)^{2}\right]^{3 / 2}} \\
& \times d z^{\prime} d x^{\prime} d y^{\prime} .
\end{aligned}
$$

Integrating out the horizontal directions $x^{\prime}$ and $y^{\prime}$ from $-\infty$ to $+\infty$ at fixed $z^{\prime}$, and integrating out the $\delta$ function, we obtain our final expression for the gravitational acceleration on the test mass

$$
\begin{aligned}
a_{\hat{m}}= & -\sum_{\vec{k}}(\hat{m} \cdot \hat{k}) 2 \pi i G \rho e^{-i \omega t} e^{-k \mathcal{H}} \\
& \times\left(\xi_{V}+\int_{0}^{\infty} \mathcal{R}\left(z^{\prime}\right) e^{-k z^{\prime}} d z^{\prime}\right) .
\end{aligned}
$$

We next solve the pendular equation of motion for the displacement $\delta \vec{x}_{j} \cdot \hat{m}_{j}$ of the test mass in response to this gravitational acceleration (where the label $j=1,2,3$, or 4 indicates which of the interferometer's four test masses we are discussing); the result is

$$
\begin{aligned}
\delta \vec{x}_{j} \cdot \hat{m}_{j}= & -\sum_{\vec{k}} \frac{\left(\hat{k} \cdot \hat{m}_{j}\right) 2 \pi i G \rho e^{i\left(\vec{k} \cdot \vec{x}_{j}-\omega t\right)} e^{-k \mathcal{H}}}{\omega_{0}^{2}-\omega^{2}-i \omega / \tau} \\
& \times\left(\xi_{V}+\int_{0}^{\infty} \mathcal{R}\left(z^{\prime}\right) e^{-k z^{\prime}} d z^{\prime}\right) .
\end{aligned}
$$

Here $\omega_{0}$ and $\tau$ are the angular eigenfrequency and damping time of the test mass's pendular motion. After completing the calculation we have moved the origin of coordinates to the interferometer's beam splitter, thereby producing the term $i \vec{k} \cdot \vec{x}_{j}$ in the exponential, where $\vec{x}_{j}$ is the test mass's location; cf. Fig. 12.

The interferometer's displacement signal $x(t)=L h(t)$ is its difference in arm lengths,

$$
x(t)=\sum_{j=1}^{4} \delta \vec{x}_{j} \cdot \hat{m}_{j}
$$

We have chosen $\hat{m}_{j}$ to point away from the test mass's mirror on the first arm and toward the mirror on the second arm as shown in Fig. 12. The seismic gravity-gradient noise is obtained by inserting expression (A13) into (A14) for each of the four test masses.

The contributions to this noise coming from the two end masses, $j=3$ and 4 , are not correlated with those coming from any other test mass in our $3-30 \mathrm{~Hz}$ frequency band, since 3 and 4 are each so far from the corner and each other $(4 \mathrm{~km} \gg \lambda=2 \pi / k)$. However, there is a significant correlation between the two corner test masses, 1 and 2. Taking account of this correlation, the interferometer's displacement

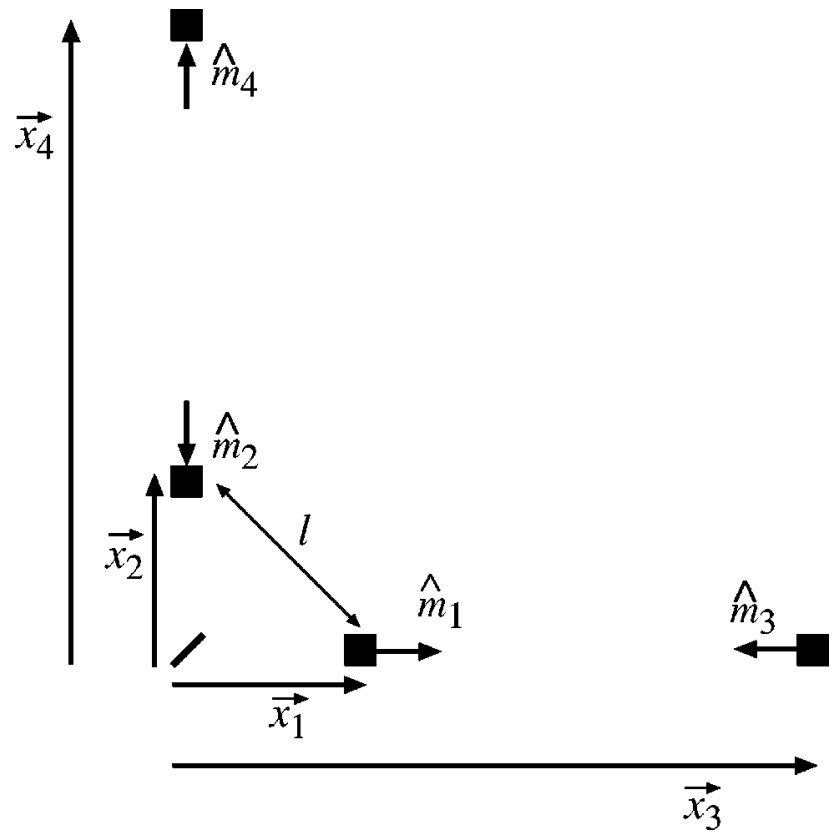

FIG. 12. The geometry of the interferometer.

signal $x(t)$ [Eqs. (A13) and (A14)] exhibits the following noise power in the frequency band $\Delta f$ :

$$
\begin{aligned}
\tilde{x}^{2}(f) \Delta f= & \frac{(2 \pi G \rho)^{2}}{\left(\omega^{2}-\omega_{0}^{2}\right)^{2}+\omega^{2} / \tau^{2}} e^{-2 k \mathcal{H}} \\
& \times \sum_{\Delta k}\left|\xi_{V}+\int_{0}^{\infty} \mathcal{R}\left(z^{\prime}\right) e^{-k z^{\prime}} d z^{\prime}\right|^{2} J_{k},
\end{aligned}
$$

where

$$
J_{k}=\sum_{\hat{k}}\left[\left|\hat{k} \cdot \hat{m}_{1} e^{i \vec{k} \cdot \vec{x}_{1}}+\hat{k} \cdot \hat{m}_{2} e^{i \vec{k} \cdot \vec{x}_{2}}\right|^{2}+\left(\hat{k} \cdot \hat{m}_{3}\right)^{2}+\left(\hat{k} \cdot \hat{m}_{4}\right)^{2}\right] \text {. }
$$

Here we have broken up the sum over $\vec{k}$ into one over all directions $\hat{k}$ and one over its length $k$ in the range $\Delta k$. Each of the last two terms in $J_{k}$ (the uncorrelated contributions of masses 3 and 4) average to $1 / 2$, and the first term can be rewritten in terms of $\vec{x}_{1}-\vec{x}_{2}$ :

$$
J_{k}=\sum_{\hat{k}}\left[\left|\hat{k} \cdot \hat{m}_{1} e^{i \vec{k} \cdot\left(\vec{x}_{1}-\vec{x}_{2}\right)}+\hat{k} \cdot \hat{m}_{2}\right|^{2}+1\right] .
$$

By virtue of the geometry of the interferometer's corner test masses (Fig. 12), $\vec{x}_{1}-\vec{x}_{2}=l\left(\hat{m}_{1}+\hat{m}_{2}\right) / \sqrt{2}$, where $l$ is the separation between those masses. Inserting this into Eq. (A17), setting $\hat{k} \cdot \hat{m}_{1}=\cos \phi$ and $\hat{k} \cdot \hat{m}_{2}=\sin \phi$, and averaging the quantity inside the sum over $\hat{k}$ (i.e., over $\phi$ ), we obtain 


$$
J_{k}=2 \sum_{\hat{k}} \gamma^{2}(k l)
$$

where

$$
\gamma(y)=\sqrt{1+\frac{1}{2 \pi} \int_{0}^{2 \pi} \cos \phi \sin \phi \cos \left(y \frac{\cos \phi+\sin \phi}{\sqrt{2}}\right) d \phi}=\sqrt{1-\frac{1}{2} J_{2}(y)}
$$

[Eq. (1.19)]. This function is graphed in Fig. 1. Inserting Eq. (A18) into Eq. (A15) and noting that $\Sigma_{\Delta k} \Sigma_{\hat{k}}=\Sigma_{\vec{k}}=N_{\Delta k}$ is the number of allowed wave vectors in the annulus $\mathcal{C}_{\Delta k}$, we obtain our final expression for the interferometer's displacement noise power:

$$
\begin{aligned}
\widetilde{x}^{2}(f) \Delta f= & \frac{(4 \pi G \rho)^{2}}{\left(\omega^{2}-\omega_{0}^{2}\right)^{2}+\omega^{2} / \tau^{2}} \\
& \times \gamma^{2}\left(\frac{\omega l}{c_{H}}\right) e^{-2 k \mathcal{H}} \mid \xi_{V}+\int_{0}^{\infty} \mathcal{R}\left(z^{\prime}\right) \\
& \times\left. e^{-k z^{\prime}} d z^{\prime}\right|^{2} \frac{N_{\Delta k}}{2} .
\end{aligned}
$$

The transfer function $T(f)$ for the seismic gravitygradient noise is obtained by dividing the direction-averaged ground displacement noise power (A8) into the interferometer displacement noise power (A20) and taking the square root. The result is expression (1.8) with the reduced transfer function $\beta$ given by $\beta=\gamma \Gamma \beta^{\prime}$ [Eq. (1.17)], where $\Gamma$ $=e^{-k \mathcal{H}}\left[\mathrm{Eq} .(1.20)\right.$ in which $\left.\omega / c_{H J}=k\right]$ and

$$
\beta^{\prime}(f)=\sqrt{\frac{3 / 2}{\left|\xi_{H}\right|^{2}+\left|\xi_{V}\right|^{2}}}\left|\xi_{V}+\int_{0}^{\infty} \mathcal{R}\left(z^{\prime}\right) e^{-k z^{\prime}} d z^{\prime}\right| ;
$$

[Eq. (1.24)].

\section{APPENDIX B: FUNDAMENTAL RAYLEIGH MODE IN HOMOGENEOUS HALF SPACE}

In this appendix we briefly review the theory of Rayleigh waves propagating in a homogeneous half space (i.e., a homogeneous, planar model of the Earth), and then we derive the anisotropy ratio $\mathcal{A}$ and reduced transfer function $\beta^{\prime}$ for such waves.

A homogeneous half space can support only the fundamental Rayleigh mode, since the overtones all require inhomogeneities to confine them in the vicinity of the Earth's surface. The theory of this mode is developed in a variety of standard texts [13-16]. According to that theory, the waves propagate with a horizontal speed $c_{H}$ which is slightly slower than the S-wave speed $c_{S}$ (which in turn is slower than $c_{P}$ ). The ratio $c_{H} / c_{S}$ is a function of the material's Poisson ratio $\nu$, varying from $c_{H} / c_{S}=0.904$ for $\nu=0.16$ (fused quartz) to $c_{H} / c_{S}=0.955$ for $\nu=0.5$ (fluids and other easily sheared materials). More generically, it is given by
$c_{H} / c_{S}=\sqrt{\zeta}$, where $\zeta$ is the real root, in the range $0<\zeta<1$, of the equation

$$
\zeta^{3}-8 \zeta^{2}+8\left(\frac{2-\nu}{1-\nu}\right) \zeta-\frac{8}{(1-\nu)}=0
$$

The Rayleigh waves' horizontal wave number is $k=\omega / c_{H}$, and their wavelength is $\lambda=2 \pi / k$. The P-wave of the fundamental Rayleigh mode decays with depth $z$ as $e^{-q k z}$, where the dimensionless ratio $q$ of vertical $e$-folding rate to horizontal wave number is

$$
q=\sqrt{1-\left(c_{H} / c_{P}\right)^{2}} .
$$

Similarly, the SV-wave part decays with depth as $e^{-s k z}$, where the dimensionless ratio $s$ of vertical $e$-folding rate to horizontal wave number is

$$
s=\sqrt{1-\left(c_{H} / c_{S}\right)^{2}}=\sqrt{1-\zeta} .
$$

More specifically, the mode's displacement eigenvector $\vec{\xi}$ can be decomposed into a P-wave which is the gradient of a scalar potential plus an SV-wave which is the curl of a vector potential. We shall denote by $\psi$ the complex amplitude of the scalar potential. The normal components of elastodynamic stress produced by this wave must vanish ${ }^{5}$ at the earth's surface. Upon imposing these boundary conditions, a standard calculation $[13,14]$ gives the following expression for the displacement vector:

$$
\begin{aligned}
\vec{\xi}= & i k \psi\left(e^{-q k z}-\frac{2 q s}{1+s^{2}} e^{-s k z}\right) \\
& \times e^{i(\vec{k} \cdot \vec{x}-\omega t)} \hat{k}-q k \psi\left(e^{-q k z}-\frac{2}{1+s^{2}} e^{-s k z}\right) \\
& \times e^{i(\vec{k} \cdot \vec{x}-\omega t)} \vec{e}_{z} .
\end{aligned}
$$

Here, $\vec{e}_{z}$ is the unit vector pointing in the $z$-direction, which we take to be down, $t$ is time, $\vec{x}$ denotes horizontal location, and $\vec{k}=k \hat{k}$ is the mode's horizontal wave vector. By comparing this displacement vector with Eq. (1.21), we read off the following expressions for the horizontal and vertical displacement amplitudes at the Earth's surface, $z=0$ :

\footnotetext{
${ }^{5}$ More accurately, they must be continuous with the stress produced by the Earth's atmosphere, which we approximate as vacuum.
} 


$$
\xi_{H}=i k \psi\left(\frac{1+s^{2}-2 q s}{1+s^{2}}\right), \quad \xi_{V}=-q k \psi\left(\frac{1-s^{2}}{1+s^{2}}\right)
$$

The wave displacement (B4) produces a fractional perturbation $\delta \rho / \rho$ of the earth's density beneath the surface given by

$$
\frac{\delta \rho(z>0)}{\rho}=-\vec{\nabla} \cdot \vec{\xi}=\mathcal{R} e^{i(\vec{k} \cdot \vec{x}-\omega t)},
$$

where

$$
\mathcal{R}(z)=\left(1-q^{2}\right) k^{2} \psi e^{-q k z} .
$$

Inserting Eqs. (B5) into Eq. (1.23), we obtain the anisotropy ratio for the $\mathrm{RF}$ mode of a homogeneous half space,

$$
\mathcal{A}=\sqrt{2} \frac{q\left(1-s^{2}\right)}{1+s^{2}-2 q s},
$$

and inserting (B5) and (B7) into (1.24) and integrating, we obtain the mode's reduced transfer function

$$
\beta^{\prime}=\sqrt{\frac{3\left(1+s^{2}-2 q\right)^{2}}{2\left(1+s^{2}\right)\left[\left(1+s^{2}\right)\left(1+q^{2}\right)-4 q s\right]}} .
$$

\section{APPENDIX C: MULTILAYER MODEL}

In this appendix we derive the equations governing Rayleigh overtones and the reduced transfer function in a multilayer model of geophysical strata.

\section{Model and notation}

Our model consists of $N$ homogeneous layers labeled by the index $n=1,2,3, \ldots, N$. Layer 1 is at the surface, layer $N$ is a homogeneous half space at the bottom, and the interfaces between layers are horizontal. The Rayleigh modes propagate as decoupled planar SV- and P-waves in each layer; they are coupled at the interfaces by continuousdisplacement and continuous-normal-stress boundary conditions.

We have already introduced much of our notation in the body of the paper; to make this appendix self-contained, we reiterate some of it here, along with some new notation:

$\omega=2 \pi f$ : Angular frequency of waves.

$\vec{k}=k \hat{k}$ : Horizontal wave vector, with $k$ its magnitude and $\hat{k}$ the unit vector in its direction.

$c_{H}=\omega / k$ : Horizontal phase velocity of waves.

$\vec{e}_{z}$ : Downward pointing unit vector.

$D_{n}$ : Thickness of layer $n$.

$z_{n}$ : Depth below the top of layer $n$.

$\vec{\xi}_{n}$ : Displacement vector for waves in layer $n$.

$K_{n}$ : Bulk modulus in layer $n$.

$\mu_{n}$ : Shear modulus in layer $n$.

$\rho_{n}$ : Density in layer $n$.

$c_{P n}$ : Speed of propagation of P-waves in layer $n$.

$c_{S n}$ : Speed of propagation of S-waves in layer $n$.

$\alpha_{P n}$ : Angle to vertical of P-wave propagation direction (between 0 and $\pi / 2$ if real, by convention). If $\mathrm{P}$-waves are evanescent in the layer, $\alpha_{P n}$ will be complex.

$\alpha_{S n}$ : Angle to vertical of SV-wave propagation vector (between 0 and $\pi / 2$ if real, by convention). If $\mathrm{SV}$-waves are evanescent in the layer, $\alpha_{S n}$ will be complex.

$\mathcal{P}_{n}$ : Complex amplitude of upgoing P-waves at the top of layer $n$.

$\mathcal{P}_{n}^{\prime}$ : Complex amplitude of downgoing P-waves at the top of layer $n$.

$\mathcal{S}_{n}$ : Complex amplitude of upgoing SV-waves at the top of layer $n$.

$\mathcal{S}_{n}^{\prime}$ : Complex amplitude of downgoing SV-waves at the top of layer $n$.

In accord with this notation, the displacement vector in layer $n$ has the following form:

$$
\begin{aligned}
& \vec{\xi}_{n}=e^{i(\vec{k} \cdot \vec{x}-\omega t)}\left[\left(\mathcal{P}_{n}^{\prime} e^{i k z_{n} \cot \alpha_{P n}}+\mathcal{P}_{n} e^{-i k z_{n} \cot \alpha_{P n}}\right) \sin \alpha_{P n} \hat{k}+\left(\mathcal{P}_{n}^{\prime} e^{i k z_{n} \cot \alpha_{P n}}-\mathcal{P}_{n} e^{-i k z_{n} \cot \alpha_{P n}}\right) \cos \alpha_{P n} \vec{e}_{z}\right. \\
& \left.+\left(\mathcal{S}_{n}^{\prime} e^{i k z_{n} \cot \alpha_{S n}}-\mathcal{S}_{n} e^{-i k z_{n} \cot \alpha_{S n}}\right) \cos \alpha_{S n} \hat{k}-\left(\mathcal{S}_{n}^{\prime} e^{i k z_{n} \cot \alpha_{S n}}+\mathcal{S}_{n} e^{-i k z_{n} \cot \alpha_{S n}}\right) \sin \alpha_{S n} \vec{e}_{z}\right] .
\end{aligned}
$$

Since the waves are generated at the Earth's surface, the upward propagating waves are absent in the lowermost layer:

$$
\mathcal{P}_{N}=0, \quad \mathcal{S}_{N}=0 .
$$

Consequently, the waves have $4 N-2$ complex amplitudes.

\section{Equations for the dispersion relation, the propagation angles, and the amplitudes}

Once one has specified the Rayleigh mode of interest, its horizontal propagation direction $\hat{k}$, and one of its amplitudes, say $\mathcal{P}_{1}$, then all its other properties are uniquely determined as a function of frequency. To evaluate its properties one first computes its horizontal dispersion relation $\omega(k)$ [or equivalently $c_{H}(f)$ ] by a procedure to be outlined below. Then one computes all the waves' propagation angles by imposing Snell's law (i.e., by demanding that all components of the wave propagate with the same horizontal speed $c_{H}$ ):

$$
\frac{c_{P n}}{\sin \alpha_{P n}}=\frac{c_{S n}}{\sin \alpha_{S n}}=c_{H} .
$$


At the Earth's surface, the (primed) amplitudes of the reflected waves are related to the (unprimed) amplitudes of the incident waves by the following two standard equations [13-16]:

$$
\begin{gathered}
2 \sin \alpha_{S 1} \cos \alpha_{P 1}\left(\mathcal{P}_{1}^{\prime}-\mathcal{P}_{1}\right)+\cos 2 \alpha_{S 1}\left(\mathcal{S}_{1}^{\prime}+\mathcal{S}_{1}\right)=0 \\
\sin \alpha_{P 1} \cos 2 \alpha_{S 1}\left(\mathcal{P}_{1}^{\prime}+\mathcal{P}_{1}\right)-\sin \alpha_{S 1} \sin 2 \alpha_{S 1}\left(\mathcal{S}_{1}^{\prime}-\mathcal{S}_{1}\right)=0 .
\end{gathered}
$$

These equations can be derived by setting the vertical-vertical and vertical-horizontal components of the stress to zero at the Earth's surface, and by expressing the ratio of bulk to shear modulus in terms of the propagation angles:

$$
\frac{K_{n}}{\mu_{n}}=\frac{c_{P n}^{2}}{c_{S n}^{2}}-\frac{4}{3}=\frac{\sin ^{2} \alpha_{P n}}{\sin ^{2} \alpha_{S n}}-\frac{4}{3} .
$$

The junction conditions at the interface between layer $n$ and layer $n+1$ take the following form [15,16]:

$$
\begin{aligned}
& \left(\mathcal{P}_{n}^{\prime} e^{i k D_{n} \cot \alpha_{P n}}+\mathcal{P}_{n} e^{-i k D_{n} \cot \alpha_{P n}}\right) \sin \alpha_{P n}+\left(\mathcal{S}_{n}^{\prime} e^{i k D_{n} \cot \alpha_{S n}}-\mathcal{S}_{n} e^{-i k D_{n} \cot \alpha_{S n}}\right) \cos \alpha_{S n} \\
& =\left(\mathcal{P}_{n+1}^{\prime}+\mathcal{P}_{n+1}\right) \sin \alpha_{P n+1}+\left(\mathcal{S}_{n+1}^{\prime}-\mathcal{S}_{n+1}\right) \cos \alpha_{S n+1},
\end{aligned}
$$

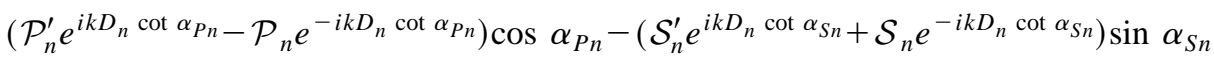

$$
\begin{aligned}
& =\left(\mathcal{P}_{n+1}^{\prime}-\mathcal{P}_{n+1}\right) \cos \alpha_{P n+1}-\left(\mathcal{S}_{n+1}^{\prime}+\mathcal{S}_{n+1}\right) \sin \alpha_{S n+1}, \\
& \mu_{n}\left[\left(1-\cot ^{2} \alpha_{S n}\right)\left(\mathcal{P}_{n}^{\prime} e^{i k D_{n} \cot \alpha_{P n}}+\mathcal{P}_{n} e^{-i k D_{n} \cot \alpha_{P n}}\right) \sin \alpha_{P n}+2\left(\mathcal{S}_{n}^{\prime} e^{i k D_{n} \cot \alpha_{S n}}-\mathcal{S}_{n} e^{-i k D_{n} \cot \alpha_{S n}}\right) \cos \alpha_{S n}\right] \\
& =\mu_{n+1}\left[\left(1-\cot ^{2} \alpha_{S n+1}\right)\left(\mathcal{P}_{n+1}^{\prime}+\mathcal{P}_{n+1}\right) \sin \alpha_{P n+1}+2\left(\mathcal{S}_{n+1}^{\prime}-\mathcal{S}_{n+1}\right) \cos \alpha_{S n+1}\right], \\
& \mu_{n}\left[2\left(\mathcal{P}_{n}^{\prime} e^{i k D_{n} \cot \alpha_{P n}}-\mathcal{P}_{n} e^{-i k D_{n} \cot \alpha_{P n}}\right) \cos \alpha_{P n}-\left(1-\cot ^{2} \alpha_{S n}\right)\left(\mathcal{S}_{n}^{\prime} e^{i k D_{n} \cot \alpha_{S n}}+\mathcal{S}_{n} e^{-i k D_{n} \cot \alpha_{S n}}\right) \sin \alpha_{S n}\right] \\
& =\mu_{n+1}\left[2\left(\mathcal{P}_{n+1}^{\prime}-\mathcal{P}_{n+1}\right) \cos \alpha_{P n+1}-\left(1-\cot ^{2} \alpha_{S n+1}\right)\left(\mathcal{S}_{n+1}^{\prime}+\mathcal{S}_{n+1}\right) \sin \alpha_{S n+1}\right] \text {. }
\end{aligned}
$$

Equation (C6a) is continuity of the horizontal displacement, (C6b) is continuity of the vertical displacement, (C6c) is continuity of the vertical-vertical component of the stress, and (C6d) is continuity of the vertical-horizontal component of the stress.

Equations (C4) and (C6a)-(C6d) are 4N-2 homogeneous linear equations for 4N-3 independent ratios of amplitudes, and for the horizontal dispersion relation $\omega(k)$ [or equivalently $c_{H}(f)$ ]. It is convenient to evaluate the dispersion relation by setting to zero the determinant of the coefficients of the amplitudes in Eqs. (C4) and (C6a) $-(\mathrm{C} 6 \mathrm{~d})$. The remaining $4 N-3$ amplitudes can then be computed in terms of $\mathcal{P}_{1}$ using any $4 N-3$ of these equations. This was the procedure used to derive the 4-layer results quoted in the text. Once the dispersion relation and the amplitudes have been evaluated as functions of frequency, the anisotropy ratio and reduced transfer function can be computed using the equations derived in the following subsection.

\section{Anisotropy ratio, and reduced transfer function}

By comparing Eq. (1.21) with the displacement eigenfunction (C1) for layer $n=1$, we read off the horizontal and vertical displacement amplitudes at the Earth's surface:

$$
\begin{aligned}
& \xi_{H}=\left(\mathcal{P}_{1}^{\prime}+\mathcal{P}_{1}\right) \sin \alpha_{P 1}+\left(\mathcal{S}_{1}^{\prime}-\mathcal{S}_{1}\right) \cos \alpha_{S 1}, \\
& \xi_{V}=-\left(\mathcal{P}_{1}^{\prime}-\mathcal{P}_{1}\right) \cos \alpha_{P 1}+\left(\mathcal{S}_{1}^{\prime}+\mathcal{S}_{1}\right) \sin \alpha_{S 1} .
\end{aligned}
$$

The wave displacement $(\mathrm{C} 1)$ produces a fractional density perturbation $\delta \rho_{n} / \rho_{n}=-\vec{\nabla} \cdot \vec{\xi}_{n}=\mathcal{R}_{n}\left(z_{n}\right) e^{i(\vec{k} \cdot \vec{x}-\omega t)}$ in layer $n$, with amplitude given by

$$
\mathcal{R}_{n}\left(z_{n}\right)=\frac{-i k}{\sin \alpha_{P n}}\left(\mathcal{P}_{n}^{\prime} e^{i k z_{n} \cot \alpha_{P n}}+\mathcal{P}_{n} e^{-i k z_{n} \cot \alpha_{P n}}\right),
$$

By inserting Eqs. (C7) and (C8) into Eq. (1.23), we obtain the anisotropy ratio

$$
\mathcal{A}=\sqrt{2}\left|\frac{\left(\mathcal{P}_{1}^{\prime}-\mathcal{P}_{1}\right) \cos \alpha_{P 1}-\left(\mathcal{S}_{1}^{\prime}+\mathcal{S}_{1}\right) \sin \alpha_{S 1}}{\left(\mathcal{P}_{1}^{\prime}+\mathcal{P}_{1}\right) \sin \alpha_{P 1}+\left(\mathcal{S}_{1}^{\prime}-\mathcal{S}_{1}\right) \cos \alpha_{S 1}}\right| .
$$

By inserting Eqs. (C7), (C8), (C9), and the relation

$$
z=z_{n}+\sum_{n^{\prime}=1}^{n-1} D_{n^{\prime}}
$$


into Eq. (1.24), integrating, and summing over all four layers, we obtain the reduced transfer function

$$
\beta^{\prime}(f)=\frac{\mathcal{N}(f)}{\mathcal{D}(f)},
$$

where

$$
\begin{aligned}
\mathcal{N}(f)= & \sqrt{\frac{3}{2}} \mid\left(\mathcal{P}_{1}-\mathcal{P}_{1}^{\prime}\right) \cos \alpha_{P}+\left(\mathcal{S}_{1}+\mathcal{S}_{1}^{\prime}\right) \sin \alpha_{S}+\sum_{n=1}^{N} \frac{\rho_{n}}{\rho_{1}}\left[-\mathcal{P}_{n} e^{i \alpha_{P n}} e^{-\left[k \Sigma_{n^{\prime}=1}^{n-1} D_{n^{\prime}}\right]}\left(1-e^{-\left[k D_{n}\left(1+i \cot \alpha_{P_{n}}\right)\right]}\right)\right. \\
& +\mathcal{P}_{n}^{\prime} e^{\left.-i \alpha_{P_{n}} e^{-\left[k \Sigma_{n^{\prime}=1}^{n-1} D_{n^{\prime}}\right]}\left(1-e^{-\left[k D_{n}\left(1-i \cot \alpha_{P_{n}}\right)\right]}\right)\right] \mid} \\
& \mathcal{D}^{2}(f)=\left|\left(\mathcal{P}_{1}^{\prime}+\mathcal{P}_{1}\right) \sin \alpha_{P 1}+\left(\mathcal{S}_{1}^{\prime}-\mathcal{S}_{1}\right) \cos \alpha_{S 1}\right|^{2}+\left|\left(\mathcal{P}_{1}^{\prime}-\mathcal{P}_{1}\right) \cos \alpha_{P 1}-\left(\mathcal{S}_{1}^{\prime}+\mathcal{S}_{1}\right) \sin \alpha_{S 1}\right|^{2}
\end{aligned}
$$

In Eq. (C12b) for $\mathcal{N}(f)$, we have inserted the factor $\rho_{n} / \rho_{1}$ to allow for the possibility (ignored in the text) that the different layers have different densities.

\section{APPENDIX D: LEE'S DISPERSION RELATION FOR 2-LAYER MODEL}

When there are only two layers, a top layer with thickness $D$ and a bottom layer with infinite thickness, the dispersion relation $\omega(k)$ [or equivalently $c_{H}(f)$ ] of the multilayer model (Appendix $C$ ) can be brought into an explicit form that permits rapid numerical solutions. This form was derived by Lee [36] by manipulating the $6 \times 6$ determinant of the coefficients of the amplitudes in Eqs. (C4) and (C6a)(C6d). The standard textbook by Eringen and Şuhubi [16] presents and discusses Lee's dispersion relation [pages 547550; note that on the first line of their Eq. (7.7.44) $\bar{\nu}_{2}$ should be $\bar{\nu}_{1}$ ]. The dispersion relation consists of the following prescription:

The unknown to be solved for is

$$
\zeta=\left(c_{H} / c_{S 2}\right)^{2} \text {. }
$$

At low propagation speeds $c_{H}$ (high frequencies) the SVwaves in layer 1 will typically propagate rather than decay, with vertical wave number divided by horizontal wave number given by

$$
\begin{aligned}
\sigma_{1} & =\sqrt{\zeta\left(c_{S 2} / c_{S 1}\right)^{2}-1}=\sqrt{\left(c_{H} / c_{S 1}\right)^{2}-1} \\
& =\cot \alpha_{S 1},
\end{aligned}
$$

while the other waves will typically be evanescent with ratios of $e$-folding rate to horizontal wave number given by

$$
\begin{aligned}
& q_{1}=\sqrt{1-\zeta\left(c_{S 2} / c_{P 1}\right)^{2}}=\sqrt{1-\left(c_{H} / c_{P 1}\right)^{2}}, \\
& q_{2}=\sqrt{1-\zeta\left(c_{S 2} / c_{P 2}\right)^{2}}=\sqrt{1-\left(c_{H} / c_{P 2}\right)^{2}}, \\
& s_{2}=\sqrt{1-\zeta}=\sqrt{1-\left(c_{H} / c_{S 2}\right)^{2}} .
\end{aligned}
$$

Regardless of the magnitude of $c_{H}$ and thence regardless of whether these quantities are real or imaginary, we regard them all as functions of $c_{H}$ given by the above expressions.

We define two quantities

$$
Q=\mu_{2} / \mu_{1}, \quad R=\rho_{1} / \rho_{2}
$$

that appear in what follows. In terms of $\zeta, Q$, and $R$, we define

$$
\begin{aligned}
& X=Q \zeta-2(Q-1), \\
& Y=Q R \zeta+2(Q-1), \\
& Z=Q(1-R) \zeta-2(Q-1), \\
& W=2(Q-1) .
\end{aligned}
$$

In this dispersion relation and only here $X, Y, Z, W$ represent these functions instead of representing Earth displacements. In terms of the above quantities we define

$$
\begin{aligned}
\xi_{1}= & \left(1-\sigma_{1}^{2}\right)\left[X \cosh \left(k q_{1} D\right)\right. \\
& \left.+\frac{q_{2}}{q_{1}} Y \sinh \left(k q_{1} D\right)\right]+2 \sigma_{1}\left[q_{2} W \sin \left(k \sigma_{1} D\right)\right. \\
& \left.-\frac{1}{\sigma_{1}} Z \cos \left(k \sigma_{1} D\right)\right] \\
\xi_{2}= & \left(1-\sigma_{1}^{2}\right)\left[s_{2} W \cosh \left(k q_{1} D\right)\right. \\
& \left.+\frac{1}{q_{1}} Z \sinh \left(k q_{1} D\right)\right]+2 \sigma_{1}\left[X \sin \left(k \sigma_{1} D\right)\right. \\
& \left.-\frac{s_{2}}{\sigma_{1}} Y \cos \left(k \sigma_{1} D\right)\right], \\
\eta_{1}= & \left(1-\sigma_{1}^{2}\right)\left[q_{2} W \cos \left(k \sigma_{1} D\right)\right. \\
& \left.+\frac{1}{\sigma_{1}} Z \sin \left(k \sigma_{1} D\right)\right]+2 q_{1}\left[-X \sinh \left(k q_{1} D\right)\right. \\
& \left.-\frac{q_{2}}{q_{1}} Y \cosh \left(k q_{1} D\right)\right],
\end{aligned}
$$




$$
\begin{aligned}
\eta_{2}= & \left(1-\sigma_{1}^{2}\right)\left[X \cos \left(k \sigma_{1} D\right)\right. \\
& \left.+\frac{s_{2}}{\sigma_{1}} Y \sin \left(k \sigma_{1} D\right)\right]+2 q_{1}\left[-s_{2} W \sinh \left(k q_{1} D\right)\right. \\
& \left.-\frac{1}{q_{1}} Z \cosh \left(k q_{1} D\right)\right]
\end{aligned}
$$

In terms of these four quantities, Lee's dispersion relation takes the form

$$
F(\zeta, k D) \equiv \xi_{1} \eta_{2}-\xi_{2} \eta_{1}=0 .
$$

In the language of Lee's dispersion relation, finding multiple Rayleigh modes is a matter of finding multiple curves $\zeta(k D)$ that satisfy (D12). Each such $\zeta(k D)$ can be translated into a corresponding $c_{H}(f)$, since $c_{H}=\sqrt{\zeta} c_{S 2}$ and $f$ $=c_{H} k / 2 \pi$. Overtone modes undergo a transition in layer 2 from propagating and lossy (so that seismic wave energy is lost from layer 1 into layer 2), to evanescent and confined (so the waves are restricted to the vicinity of the top layer) at speed $c_{H}(f)=c_{S 2}$, which is equivalent to $\zeta=1$. Thus, to produce dispersion relations for overtone modes, one can look for solutions to (D12) in the vicinity of $\zeta=1$, and then, depending on whether one wants confined modes or lossy modes, trace them from $\zeta=1$ to higher frequencies and lower horizontal speeds, or to lower frequencies and higher horizontal speeds.

In Sec. IV C 3 we use Lee's dispersion relation to study the RP1 mode at Livingston in the lossy regime.

\section{APPENDIX E: P-UP AND SV-UP MODES}

In the text we encounter situations in which one can approximate an overtone mode as $\mathrm{P}$ - or $\mathrm{SV}$-waves that propagate upward through a homogeneous half space until they encounter the Earth's surface or one or more layers near the surface, and then (exciting the layers) reflect back downward with accompanying production of the other type of wave. Such "P-up" and "SV-up" modes can be described by the multilayer equations of Appendix $\mathrm{C}$, with the up (unprimed) amplitudes in the bottom layer (homogeneous half space), $b$, set to $\left\{\mathcal{P}_{b} \neq 0, \mathcal{S}_{b}=0\right\}$ for P-up modes, and $\left\{\mathcal{P}_{b}=0, \mathcal{S}_{b} \neq 0\right\}$ for SV-up modes.

We can derive simple formulas for the anisotropy ratio $\mathcal{A}$ and reduced transfer function $\beta^{\prime}$ of such modes for the case of no surface layers (a pure homogeneous half space).

\section{P-up modes in a homogeneous half space}

The displacement function is given by Eq. (C1) with the subscript $n$ 's deleted since there is only one layer. The primed (down) amplitudes are given in terms of the unprimed (up) amplitude $\mathcal{P}$ by the surface junction conditions (C4); in particular

$$
\mathcal{P}^{\prime}=\frac{4 \cos \alpha_{P} \sin ^{3} \alpha_{S} \cos \alpha_{S}-\sin \alpha_{P} \cos ^{2} 2 \alpha_{S}}{4 \cos \alpha_{P} \sin ^{3} \alpha_{S} \cos \alpha_{S}+\sin \alpha_{P} \cos ^{2} 2 \alpha_{S}} \mathcal{P},
$$

$$
\mathcal{S}^{\prime}=\frac{4 \sin \alpha_{P} \cos \alpha_{P} \sin \alpha_{S} \cos 2 \alpha_{S}}{4 \cos \alpha_{P} \sin ^{3} \alpha_{S} \cos \alpha_{S}+\sin \alpha_{P} \cos ^{2} 2 \alpha_{S}} \mathcal{P} .
$$

Inserting these into Eq. (C10) we obtain the following anisotropy ratio:

$$
\mathcal{A}=\sqrt{2} \cot 2 \alpha_{S},
$$

where, by Snell's law [Eq. (3.1)],

$$
\alpha_{S}=\arcsin \left(c_{S} / c_{H}\right)
$$

Inserting expressions (E1) into the one-layer version of equations (C12), we obtain the following reduced transfer function:

$$
\beta^{\prime}=\sqrt{6} \sin ^{2} \alpha_{S}
$$

The anisotropies and reduced transfer functions of Eqs. (E2) and (E4) are shown graphically in Figs. 3 and 4 for $c_{P} / c_{S}$ $=2$ (approximately appropriate to the surface materials at Livingston and Hanford).

\section{SV-up modes in a homogeneous half space}

For SV-up modes, as for P-up modes, the displacement function is given by Eq. (C1) with the subscript $n$ 's deleted. The primed (down) amplitudes are given in terms of the unprimed (up) amplitude $\mathcal{S}$ by the surface junction conditions (C4); in particular

$$
\begin{aligned}
& \mathcal{P}^{\prime}=-\frac{\sin \alpha_{S} \sin 4 \alpha_{S}}{4 \cos \alpha_{P} \sin ^{3} \alpha_{S} \cos \alpha_{S}+\sin \alpha_{P} \cos ^{2} 2 \alpha_{S}} \mathcal{S}, \\
& \mathcal{S}^{\prime}=\frac{4 \cos \alpha_{P} \sin ^{3} \alpha_{S} \cos \alpha_{S}-\sin \alpha_{P} \cos ^{2} 2 \alpha_{S}}{4 \cos \alpha_{P} \sin ^{3} \alpha_{S} \cos \alpha_{S}+\sin \alpha_{P} \cos ^{2} 2 \alpha_{S}} \mathcal{S}
\end{aligned}
$$

Inserting these into Eq. (C10), we obtain the following anisotropy ratio

$$
\mathcal{A}=2 \sqrt{2}\left|\frac{\cot \alpha_{P}}{\cot ^{2} \alpha_{S}-1}\right|,
$$

where, by Snell's law,

$$
\alpha_{S}=\arcsin \left(c_{S} / c_{H}\right), \alpha_{P}=\arcsin \left(c_{P} / c_{H}\right) .
$$

Inserting expressions (E5) into the one-layer version of equations (C12), we obtain the following reduced transfer function:

$$
\beta^{\prime}=\frac{\sqrt{6} \sin ^{2} \alpha_{S}\left|1-2 i \cot \alpha_{P} \sin ^{2} \alpha_{S} \sec 2 \alpha_{S}\right|}{\sqrt{1+\left(2\left|\cot \alpha_{P}\right| \sin ^{2} \alpha_{S} \sec 2 \alpha_{S}\right)^{2}}} .
$$

The anisotropies and reduced transfer functions of Eqs. (E6) and (E8) are shown in Figs. 3 and 4, for $c_{P} / c_{S}=2$. 
[1] A. Abramovici et al., Science 256, 325 (1992); see also http:// www.ligo.caltech.edu/.

[2] B. Caron et al., Class. Quantum Grav. 14, 1461 (1997); see also http://www.pg.infn.it/virgo/.

[3] J. W. Cronin et al., in Proceedings of the Snowmass 95 Summer Study on Particle and Nuclear Astrophysics, edited by E. W. Kolb and R. Peccei (World Scientific, Singapore, 1995).

[4] K. S. Thorne, in Black Holes and Relativistic Stars, edited by R. M. Wald (University of Chicago Press, Chicago, 1998), p. 41, gr-qc/9706079.

[5] K. S. Thorne and C. J. Winstein, gr-qc/9810016.

[6] S. Braccini et al., Rev. Sci. Instrum. 64, 310 (1993); M. Beccaria et al., Nucl. Instrum. Methods Phys. Res. A 394, 397 (1997).

[7] R. Weiss, “Quarterly Progress Report of the Research Laboratory of Electronics of the Massachusetts Institute of Technology"' (1972), Vol. 105, p. 54.

[8] P. R. Saulson, Phys. Rev. D 30, 732 (1984).

[9] R. Spero, in Science Underground, Proceedings of the Los Alamos Conference, 1982, edited by M. M. Nieto et al. (AIP, New York, 1983).

[10] P. R. Saulson, Fundamentals of Interferometric Gravitational Wave Detectors (World Scientific, Singapore, 1994).

[11] S. Bracini et al., Class. Quantum. Grav. (to be published). The corresponding authors for this 42-author paper are G. Cella and E. Cuoco.

[12] S. A. Hughes and K. S. Thorne (unpublished).

[13] L. D. Landau and E. M. Lifshitz, Theory of Elasticity (Pergamon, Oxford, 1986), Chap. 24.

[14] A. E. H. Love, A Treatise on the Mathematical Theory of Elasticity, 4th ed. (Dover, New York, 1944).

[15] W. L. Pilant, Elastic Waves in the Earth (Elsevier Scientific Publishing Company, New York, 1979), Chap. 8.

[16] A. C. Eringen and E. S. Suhubi, Elastodynamics, Volume II, Linear Theory (Academic, New York, 1975), Chap. 7.

[17] R. E. Abercrombie, Bull. Seismol. Soc. Am. 87, 731 (1997); also, for a review of measurements: R. E. Abercrombie, Pure Appl. Geophys., special volume, edited by B. J. Mitchell and B. A. Romanovicz (unpublished).

[18] K. Ishihara, Soil Behavior in Earthquake Geotechnics (Oxford Science Publications, Oxford, 1996).
[19] CRC Practical Handbook of Physical Properties of Rocks and Minerals, edited by R. S. Carmichael (CRC, Boca Raton, FL, 1989).

[20] A. Rohay, "Ambient Ground Vibration Measurements at the Livingston, Louisiana LIGO Site,' LIGO Document LIGOC961022-A-O1.

[21] A. Rohay, "Ambient Ground Vibration Measurements at the Hanford, Washington LIGO Site,' LIGO Document LIGOC950572-02-O1.

[22] M. W. Asten, Bull. Seismol. Soc. Am. 68, 1623 (1978).

[23] E. J. Douze and S. J. Laster, Geophysics 44, 1570 (1979).

[24] A. C. Liaw and T. V. McEvilly, Geophysics 44, 1097 (1979).

[25] C. A. Powell, Bull. Seismol. Soc. Am. 82, 1889 (1992).

[26] S. E. Hough et al., Bull. Seismol. Soc. Am. 82, 1186 (1992).

[27] G. Milana et al., Bull. Seismol. Soc. Am. 86, 320 (1996).

[28] E. J. Douze, Bull. Seismol. Soc. Am. 57, 55 (1967).

[29] A. Rohay, "Analysis of Differential Motions at the Hanford, Washington and Livingston, Louisiana LIGO Sites,' LIGO Document LIGO-C962289-B-01.

[30] A. Rohay (private communication).

[31] K. S. Thorne, in 300 Years of Gravitation, edited S. W. Hawking and W. Israel (Cambridge University Press, Cambridge, England, 1987).

[32] "Report of Geotechnical Survey," LIGO Project, Hanford, Washington, Dames and Moore, 1993.

[33] "Safety Evaluation Report Related to the Operation of WPPS Nuclear Project No. 2,' Report NUREG-0892 (Nuclear Regulatory Commission, Washington DC, 1982), Sec. 2.5, dated $12 / 21 / 81$.

[34] "Geotechnical Investigation of the LIGO site," Livingston, Louisiana, Woodward-Clyde Consultants, 1995.

[35] H. Liu et al., Engineering Geology 46, 313 (1997).

[36] A. W. Lee, Mon. Not. R. Astron. Soc. Geophys. Suppl. 3, 83 (1932).

[37] LIGO site blueprints, Document Numbers LIGO-D960052O0- $\square, \quad$ LIGO-D960055-O0- $\square ， \quad$ LIGO-D960917-O0- $\square$, LIGO-D960242-A- $\square$.

[38] L. Malagnini, A. Rovelli, S. E. Hough, and L. Seeber, Bull. Seismol. Soc. Am. 83, 1744 (1993)

[39] Hiroo Kanamori (private communication).

[40] Dilatometers are described in I. S. Sacks et al., Pap. Meteorol. Geophys. 23, 195 (1971). 